\title{
CANONICAL PSEUDOTENSORS, SPARLING'S FORM AND NOETHER CURRENTS
}

\author{
L. B. Szabados \\ Central Research Institute for Physics \\ H - 1525 Budapest 114, P.O.Box 49, Hungary
}

\begin{abstract}
The Lagrangian scenario is applied both to Einstein's first and Hilbert's second order Lagrangians when the field variable is the metric and when it is a rigid basis of 1-forms. The canonical spin pseudotensors, as the gravitational counterparts of the canonical spin tensor of the matter fields, are introduced and it is shown that they play the role of superpotentials for the canonical energy-momentum pseudotensors. The canonical spin pseudotensors distinguishes von Freud's and Goldberg's superpotentials for Einstein's, and Møller's superpotential for Hilbert's Lagrangian. (For Hilbert's Lagrangian there is no difference between the metric and rigid basis descriptions.) Møller's energy-momentum pseudotensor is therefore recovered as the canonical energy-momentum pseudotensor for Hilbert's Lagrangian. The contravariant form of the canonical pseudotensors are shown to satisfy Belinfante-Rosenfeld type equations, furthermore their Belinfante-Rosenfeld combination is always the Einstein tensor. It is shown that, for first order Lagrangian and rigid basis description, the canonical energy-momentum, the canonical spin and the Noether current are tensorial quantities, and the canonical energy-momentum and spin tensors satisfy the tensorial Belinfante-Rosenfeld equations. These tensorial quantities, however, depend on the basis of 1forms we use.

The second part of the paper is the differential geometric unification and reformulation of the previous different pseudotensorial approaches. First it is shown that, along coordinate sections, the pull backs of the contravariant and dual forms of Sparling's form, defined on the bundle of linear frames $L(M)$ over the $m$ dimensional spacetime, are the Bergmann and the Landau-Lifshitz pseudotensors, respectively. Although the pull backs of Sparling's form along rigid sections are not exactly the energy-momentum tensors, they are always tensorial and the pull backs of the full Sparling equation are always the equations expressing the canonical (pseudo) tensors by the corresponding superpotentials. A $g l(m, \mathbf{R})$ valued $(m-1)$ form, called the spin form, is defined on $L(M)$, and it is shown that its pull backs are the various canonical spin (pseudo) tensors. An exterior differential equation for the contravariant form of the spin form is derived, whose pull backs are just the Belinfante-Rosenfeld equations for the canonical (pseudo) tensors. In terms of the spin form a necessary and sufficient condition is found for the metric connections being torsion free and to satisfy Einstein's equations. Finally, for any vector field on the spacetime an $(m-1)$ form, called the Noether form, is defined on $L(M)$ whose pull backs to the spacetime are the corresponding Noether (pseudo) currents.
\end{abstract}




\section{Introduction}

In special relativistic classical Lagrangian field theories one can associate ten conserved quantities, namely divergence free vector fields, to the ten independent Killing vectors of Minkowski's spacetime [1]. As a consequence of the semi-direct sum structure of the Lie algebra of the Killing vectors it is natural to interpret the conserved quantities as the linear and angular momenta of the matter fields. If the spacetime is curved but it is of constant curvature then the Lie algebra of the Killing vectors is so $(1,4)$ or $s o(2,3)$. Thus although one has ten conserved quantities again, their interpretation as linear and angular momenta is not obvious, because both $s o(1,4)$ and $s o(2,3)$ are semisimple and do not admit canonical " $4+6$ " decomposition. If the spacetime is not of constant curvature then the number of independent Killing vectors, and hence the number of conserved kinematical quantities, is less than ten, e.g. zero; and, apart from exceptional cases, their interpretation is far from being obvious.

In a pure geometric interpretation of gravity [2] the result is not surprising in the light of the first Noether theorem: if the spacetime does not admit symmetries then one cannot expect to have conserved kinematical quantities. In the field theoretical interpretation of gravity [3] one can say that the matter fields together is not a closed system, they are interacting with the gravitational field and hence the kinematical quantities of the matter fields are not constant. One can have conserved kinematical quantities for the matter fields only if the "gravitational interaction" has symmetries, i.e. Killing vectors. Since, however, the matter and gravitational fields together can be considered as a semi - closed system (i.e. a closed system allowing in- and out going radiation), one may expect to be able to associate kinematical quantities to the gravitational field such that they, together with those of the matter fields, be conserved [4-8]. In fact, this philosophy works for non - gravitational matter fields on Minkowski's spacetime, where one has ten independent Killing symmetries as symmetries of the whole material action too, and hence, using the first Noether theorem, one can construct the conserved currents to the Killing symmetries [4-8]. Although these conserved quantities are unique only up to a curl, there is a canonical choice to build up these currents from the canonical energy - momentum and spin tensors of the matter fields and the Killing vectors. These are the canonical Noether currents. However, in the case of gravitation one does not have (ten) Killing vectors and hence one cannot use the first Noether theorem to construct the (ten) gravitational kinematical quantities. One has too few symmetries. On the other hand any smooth vector field generates a 1 parameter group of coordinate transformations leaving the field equations invariant and forming a subgroup of the group of general coordinate transformations. As a consequence of the two Noether theorems one has a weak and a strong conservation law [4-10]. Unfortunately, the conserved quantity is not determined uniquely, one can add to it a curl, and it does not seem to have a canonical choice for the "currents". Because of the arbitrariness of the vector field, the interpretation of the conserved quantity is far from being obvious. Now one has too many "symmetries". The most serious difficulty is, however, the non tensorial character of both these conserved quantities and the conservation equations. One of the most important achievements of general relativity is probably the perception that Nature must be described in terms of absolute elements; i.e. geometric objects ("principle of general covariance"). Thus the usage of these non tensorial quantities seems to contradict to the principle of general covariance, and, as is written in [29], this "difficulty has led most physicists to the conclusion that the problem is ill-defined and the field irrelevant, density of gravitational energy is like density of beauty of a panting". Nevertheless, in certain situations, e.g. in studying the gravitational 
radiation, gravitational energy - momentum and angular - momentum like quantities would be useful.

The present paper, which is intended to be the first part of a series, is devoted to the problem of linear and angular momenta in general relativity. First we consider the "orthodox" pseudotensorial description [4-10]. To choose from the mathematically possible infinitely many different pseudotensors we follow the scenario of the Lagrangian formalism of fields, where we have not only the canonical energy - momentum, but the canonical spin tensor as well [10-15]. The most important characteristic feature of these canonical tensors is the pair of Belinfante - Rosenfeld relations for them. In paragraph 2.1. this formalism is reviewed for tensorial matter fields and second order Lagrangian. The Belinfante - Rosenfeld combination of the canonical tensors, the Noether currents and the invariance properties of these objects are also considered.

In paragraph 2.2. the canonical energy - momentum and spin pseudotensors are defined for second order Lagrangians and tensorial gravitational field variables. The notion of spin pseudotensor introduced here is believed to be new. In paragraph 2.3. and 2.4. the general scenario is applied for Einstein's first order Lagrangian first if the gravitational field variable is the metric and then if it is a rigid basis of 1 forms. The canonical pseudotensors for Hilbert's second order Lagrangian are considered in paragraph 2.5., and finally, in paragraph 2.6., the Landau - Lifshitz - Goldberg type (non canonical) pseudotensors, appearing naturally as Belinfante - Rosenfeld combinations, are considered.

The appearing contradiction between these canonical gravitational quantities and the principle of general covariance has not been resolved. To do this one should try to find geometric objects describing the energy - momentum and spin content of gravity. In the second part of the present paper we deal with certain differential forms defined on the bundle of linear frames, the Sparling form $[28,29]$ and a so-called spin form, that we can interpret as the geometric objects we search for. In paragraph 3.1. the differential geometric formulae we need are reviewed. Paragraph 3.2. is devoted to the study of Sparling's form. The pull back of this form along a coordinate section is known to be Einstein's canonical energy - momentum pseudotensor [32,33]. Here the relation between Sparling's form and other energy - momentum pseudotensors is clarified. In paragraph 3.3. the spin form is introduced, Belinfante - Rosenfeld type equation for the spin and energy momentum forms are derived and the relation between the spin form and the spin pseudotensors is clarified. Then the Noether form is introduced on the bundle of linear frames and its pull backs along various sections of the bundle are shown to be the various Noether pseudocurrents built up from the canonical pseudotensors. Finally, in the last section of this paper we summarize and discuss the results. In our following paper we will deal with the quasi local integrals of the pull back of the Noether form, their interpretation and properties [34].

Since the aim of the present paper is not only to derive new formulae for new (and old) pseudotensorial quantities, but also to study the structure of these equations to be able to reformulate them in the differential geometric language, some formulae will be known from the pseudotensor literature. (Thus the first part of this paper may also be considered as a review of the canonical pseudotensors from a specific point of view, but it is definitely more than a pure review.) But this literature is so extensive $[4-10,18-23,25,26]$ that it is almost impossible to cite always the original papers where they were published. Thus we frequently refer only to widely accepted and well known review articles [4-6]. 


\section{Canonical pseudotensors of Einstein's theory}

\subsection{Canonical energy-momentum and spin tensors of matter fields}

Let $M$ be an $m$ dimensional manifold, $g$ a metric on $M$ of signature $p-q, p+q=m$, let $\nabla_{\mu}$ be the unique torsion-free covariant derivation determined by $g$ and $\varepsilon$ the natural volume $m$-form associated to $g$; i.e. if $\left(x^{1}, \ldots, x^{m}\right)$ is a local coordinate system then $\varepsilon=\sqrt{|g|} \epsilon_{\alpha_{1} \ldots \alpha_{m}} d x^{\alpha_{1}} \wedge \ldots \wedge d x^{\alpha_{m}}$ $=m ! \sqrt{|g|} d x^{1} \wedge \ldots \wedge d x^{m}$. $\left(\epsilon_{\alpha_{1} \ldots \alpha_{m}}\right.$ is the totally skew Levi-Civita symbol, $\epsilon_{1 \ldots m}=1$, Greek indexes are coordinate indexes and for the exterior product the convention compatible with $2 d x^{1} \wedge d x^{2}=$ $d x^{1} \otimes d x^{2}-d x^{2} \otimes d x^{1}$ is used. Abstract indexes will not be used in this paper.)

By matter fields we mean a finite $N$ number of tensor fields $\Phi_{\beta_{1} \ldots \beta_{s}}^{n \alpha_{1} \ldots \alpha_{r}}, n=1, \ldots, N$, and the type $(r, s)$ of the $n$th field may depend on $n$. The Lagrangian for these fields is assumed not to depend on higher than the second covariant derivatives of the fields: $L=L_{m}\left(g_{\alpha \beta}, \Phi_{\beta_{1} \ldots \beta_{s}}^{n \alpha_{1} \ldots \alpha_{r}}\right.$, $\nabla_{\mu} \Phi_{\beta_{1} \ldots \beta_{s}}^{n \alpha_{1} \ldots \alpha_{r}}, \nabla_{\mu} \nabla_{\nu} \Phi_{\beta_{1} \ldots \beta_{s}}^{n \alpha_{1} \ldots \alpha_{r}}$ ). If no confusion can arise we will frequently omit the spacetime indexes of the matter fields. For an open domain $D \subset M$ having compact closure and a field configuration $\Phi^{n}(x)$ the action functional is defined as $I_{D}[g, \Phi]:=\int_{D} L\left(g_{\alpha \beta}(x), \Phi^{n}(x), \nabla_{\mu} \Phi^{n}(x)\right.$, $\left.\nabla_{\mu} \nabla_{\nu} \Phi^{n}(x)\right) \varepsilon$. The Euler-Lagrange expressions for the matter fields (i.e. the functional derivative of $I_{D}[g, \Phi]$ with respect to $\left.\Phi^{n}\right)$ are

$$
E_{n}[L, \Phi]:=\frac{\partial L}{\partial \Phi^{n}}-\nabla_{\mu}\left(\frac{\partial L}{\partial \nabla_{\mu} \Phi^{n}}\right)+\nabla_{\mu} \nabla_{\nu}\left(\frac{\partial L}{\partial \nabla_{\nu} \nabla_{\mu} \Phi^{n}}\right) .
$$

Let $r, s$ be fixed nonnegative integers. For $r=s=0$ let $\Delta_{\nu}^{\mu}=0$, while for $r+s \neq 0$ let us define

$$
\begin{aligned}
\Delta_{\nu \beta_{1} \ldots \beta_{s} \gamma_{1} \ldots \gamma_{r}}^{\mu \alpha_{1} \ldots \alpha_{r} \delta_{1} \ldots \delta_{s}} & =\left(\delta_{\nu}^{\alpha_{1}} \delta_{\gamma_{1}}^{\mu} \ldots \delta_{\gamma_{r}}^{\alpha_{r}}+\ldots+\delta_{\gamma_{1}}^{\alpha_{1}} \ldots \delta_{\nu}^{\alpha_{r}} \delta_{\gamma_{r}}^{\mu}\right) \delta_{\beta_{1}}^{\delta_{1}} \ldots \delta_{\beta_{s}}^{\delta_{s}}- \\
& \left.-\delta_{\gamma_{1}}^{\alpha_{1} \ldots \delta_{\gamma_{r}}} \alpha_{\nu}^{\alpha_{r}} \delta_{\beta_{1}}^{\delta_{1}} \delta_{\beta_{s}}^{\mu}+\ldots+\delta_{\beta_{1}}^{\delta_{s}} \ldots \delta_{\nu}^{\delta_{s}} \delta_{\beta_{s}}^{\mu}\right)
\end{aligned}
$$

This is just the invariant tensor occurring in the expression of the Lie derivative $\mathrm{E}_{\mathbf{K}} \Phi$ of an $(r, s)$ type tensor field $\Phi$ along the vector field $\mathbf{K}$ in terms of the covariant derivatives:

$$
\mathrm{E}_{\mathbf{K}} \Phi_{\beta_{1} \ldots \beta_{s}}^{\alpha_{1} \ldots \alpha_{r}}=\nabla_{\mathbf{K}} \Phi_{\beta_{1} \ldots \beta_{s}}^{\alpha_{1} \ldots \alpha_{r}}-\nabla_{\mu} K^{\nu} \Delta_{\nu \beta_{1} \ldots \beta_{s} \gamma_{1} \ldots \gamma_{r}}^{\mu \alpha_{1} \ldots \alpha_{r} \delta_{1} \ldots \delta_{s}} \Phi_{\delta_{1} \ldots \delta_{s}}^{\gamma_{1} \ldots \gamma_{r}}
$$

Since the first term on the right hand side contains $\mathbf{K}$ purely algebraically while the second is proportional to the first derivative of $\mathbf{K}$, this expression may be interpreted as the splitting up of the rate of the Lie dragging along $\mathbf{K}$ into a translational and a rotational part. (Similarly to the spacetime indexes of the matter fields the $r+s$ upper and $r+s$ lower indexes of $\Delta_{\nu \beta_{1}, \ldots, \beta_{s} \gamma_{1} \ldots \delta_{r}}^{\mu \alpha_{1} \ldots \delta_{2} \delta_{1} \ldots \delta_{1}}$ not in general be written out. Thus, for example, $\Delta_{\nu}^{\mu} \nabla_{\rho} \Phi$ will stand for $\Delta_{\nu \beta_{1} \ldots \beta_{s} \gamma_{1} \ldots \gamma_{r}}^{\mu \alpha_{1} \ldots \alpha_{\rho} \delta_{1} \ldots \delta_{s}} \nabla_{\delta_{1} \ldots \delta_{s}}^{\gamma_{1} \ldots \gamma_{r}}$.) By means of $\Delta_{\nu}^{\mu}$ the commutators of the covariant derivations and the covariant and Lie derivations can be written in the following short forms:

$$
\left(\nabla_{\alpha} \nabla_{\beta}-\nabla_{\beta} \nabla_{\alpha}\right) \Phi=R_{\mu \alpha \beta}^{\nu} \Delta_{\nu}^{\mu} \Phi
$$

and

$$
\mathrm{E}_{\mathbf{K}} \nabla_{\alpha} \Phi-\nabla_{\alpha} \mathrm{E}_{\mathbf{K}} \Phi=\left(-R_{\mu \alpha \beta}^{\nu} K^{\beta}+\nabla_{\alpha} \nabla_{\mu} K^{\nu}\right) \Delta_{\nu}^{\mu} \Phi
$$

(Eq. (2.1.4.) shows that our sign convention for the curvature tensor is the same that is used in $[2,27]$. The Ricci tensor is defined by $R_{\alpha \beta}:=R_{\alpha \mu \beta}^{\mu}$.)

It will be useful to introduce the following tensor field: 


$$
\begin{aligned}
\sigma_{\beta}^{\mu \alpha}: & =\left(\frac{\partial L}{\partial \nabla_{\mu} \Phi^{n}}-\nabla_{\nu}\left(\frac{\partial L}{\partial \nabla_{\nu} \nabla_{\mu} \Phi^{n}}\right)\right) \Delta_{\beta}^{\alpha} \Phi^{n}+ \\
& +\frac{\partial L}{\partial \nabla_{\mu} \nabla_{\nu} \Phi^{n}}\left(\Delta_{\beta}^{\alpha} \nabla_{\nu} \Phi^{n}-\delta_{\nu}^{\alpha} \nabla_{\beta} \Phi^{n}\right) .
\end{aligned}
$$

Using this definition, the dynamical (or symmetric) energy-momentum tensor (which is, by definition, twice the functional derivative of $I_{D}[g, \Phi]$ with respect to $g_{\alpha \beta}$ ) takes the form:

$$
\begin{aligned}
T^{\alpha \beta} & =2 \frac{\partial L}{\partial g_{\alpha \beta}}+L g^{\alpha \beta}+ \\
& +\frac{1}{2} \nabla_{\mu}\left(\sigma^{\alpha \beta \mu}+\sigma^{\beta \alpha \mu}-\sigma^{\alpha \mu \beta}-\sigma^{\beta \mu \alpha}-\sigma^{\mu \alpha \beta}-\sigma^{\mu \beta \alpha}\right) .
\end{aligned}
$$

Let $\mathbf{K}$ be any vector field on $M$. Then

$$
\begin{aligned}
\nabla_{\mu}\left(L K^{\mu}\right) & =\mathrm{L}_{\mathbf{K}} L+\frac{1}{2} L g^{\alpha \beta} \mathrm{L}_{\mathbf{K}} g_{\alpha \beta}= \\
& =\left(\frac{\partial L}{\partial g_{\alpha \beta}}+\frac{1}{2} L g^{\alpha \beta}\right) \mathrm{E}_{\mathbf{K}} g_{\alpha \beta}+E_{n}[L, \Phi] \mathrm{E}_{\mathbf{K}} \Phi^{n}+ \\
& +\nabla_{\mu}\left(\left[\frac{\partial L}{\partial \nabla_{\mu} \Phi^{n}}-\nabla_{\nu}\left(\frac{\partial L}{\partial \nabla_{\nu} \nabla_{\mu} \Phi^{n}}\right)\right] \mathrm{E}_{\mathbf{K}} \Phi^{n}+\frac{\partial L}{\partial \nabla_{\mu} \nabla_{\nu} \Phi^{n}} \mathrm{~L}_{\mathbf{K}} \nabla_{\nu} \Phi^{n}\right)+ \\
& +\left(\frac{\partial L}{\partial \nabla_{\mu} \Phi^{n}}-\nabla_{\nu}\left(\frac{\partial L}{\partial \nabla_{\nu} \nabla_{\mu} \Phi^{n}}\right)\right)\left\{\mathrm{L}_{\mathbf{K}} \nabla_{\mu} \Phi^{n}-\nabla_{\mu} \mathrm{E}_{\mathbf{K}} \Phi^{n}\right\}+ \\
& +\frac{\partial L}{\partial \nabla_{\mu} \nabla_{\nu} \Phi^{n}}\left\{\mathrm{~L}_{\mathbf{K}} \nabla_{\mu} \nabla_{\nu} \Phi^{n}-\nabla_{\mu} \mathrm{L}_{\mathbf{K}} \nabla_{\nu} \Phi^{n}\right\}
\end{aligned}
$$

Let us define

$$
\begin{aligned}
\theta_{\nu}^{\mu}:=L \delta_{\nu}^{\mu} & -\left(\frac{\partial L}{\partial \nabla_{\mu} \Phi^{n}}-\nabla_{\rho}\left(\frac{\partial L}{\partial \nabla_{\rho} \nabla_{\mu} \Phi^{n}}\right)\right) \nabla_{\nu} \Phi^{n}- \\
& -\frac{\partial L}{\partial \nabla_{\mu} \nabla_{\rho} \Phi^{n}} \nabla_{\nu} \nabla_{\rho} \Phi^{n} .
\end{aligned}
$$

Using the definitions of $\sigma^{\mu \alpha}{ }_{\beta}$ and ${\theta^{\mu}}_{\nu}$, the expression (2.1.7.) for $T^{\alpha \beta}$ and (2.1.4.) and (2.1.5.) both for the $(r, s)$ and $(r, s+1)$ type fields $\Phi$ and $\nabla_{\nu} \Phi$, after a rather long calculation one arrives at

$$
\begin{aligned}
\nabla_{\mu}\left(L K^{\mu}\right) & =E_{n}[L, \Phi] \mathrm{E}_{\mathbf{K}} \Phi^{n}+\frac{1}{2} T^{\alpha \beta} \mathrm{Ł}_{\mathbf{K}} g_{\alpha \beta}+ \\
& +\nabla_{\mu}\left(L K^{\mu}-\theta^{\mu}{ }_{\nu} K^{\nu}-\left(\sigma^{\mu[\alpha \beta]}+\sigma^{\alpha[\beta \mu]}+\sigma^{\beta[\alpha \mu]}\right) \nabla_{\alpha} K_{\beta}\right)
\end{aligned}
$$

and hence

$$
E_{n}[L, \Phi] \mathrm{L}_{\mathbf{K}} \Phi^{n}+\frac{1}{2} T^{\alpha \beta} \mathrm{L}_{\mathbf{K}} g_{\alpha \beta}=\nabla_{\mu}\left(\theta_{\nu}^{\mu} K^{\nu}+\left(\sigma^{\mu[\alpha \beta]}+\sigma^{\alpha[\beta \mu]}+\sigma^{\beta[\alpha \mu]}\right) \nabla_{\alpha} K_{\beta}\right) .
$$

The most important consequences of this identity are the following [11-15]:

1. For the field configurations satisfying the Euler-Lagrange equations, $E_{n}[L, \Phi]=0, T^{\alpha \beta}$ is divergence free: $\nabla_{\alpha} T^{\alpha \beta}=0$. 
2. Using $\nabla_{\alpha} T^{\alpha \beta}=0$ and $E_{n}[L, \Phi]=0$ again, one obtains:

$$
T^{\alpha \beta}=\theta^{\alpha \beta}+\nabla_{\mu}\left(\sigma^{\mu[\alpha \beta]}+\sigma^{\alpha[\beta \mu]}+\sigma^{\beta[\alpha \mu]}\right)
$$

We will call its right hand side as the Belinfante-Rosenfeld combination of $\theta^{\alpha}{ }_{\beta}$ and $\sigma^{\mu \alpha}{ }_{\beta}$. Taking its antisymmetric part and its divergence, respectively, one has

$$
\theta^{[\alpha \beta]}=-\nabla_{\mu} \sigma^{\mu[\alpha \beta]}
$$

and

$$
\nabla_{\mu} \theta_{\nu}^{\mu}=-R_{\nu \rho \alpha \beta} \sigma^{\rho \alpha \beta}
$$

These will be called the algebraic and differential Belinfante-Rosenfeld relations, respectively.

If $\mathbf{K}$ is any smooth vector field and $\psi_{t}$ is the local 1 parameter transformation group generated by $\mathbf{K}$ such that $\psi_{0}=I d$ then $\left(\frac{d}{d t} I_{\psi_{t}(D)}[g, \Phi]\right)_{t=0}=-\int_{D} \mathrm{E}_{\mathbf{K}}(L \varepsilon)=-\int_{D} \nabla_{\mu}\left(L K^{\mu}\right) \varepsilon$. Thus if $\mathbf{K}$ is a Killing vector and the Euler-Lagrange equations are satisfied then the infinitesimal changing of the action integral along $\mathbf{K}$ is only a surface term. Its translational part is connected to $L \delta_{\nu}^{\mu}-\theta^{\mu}{ }_{\nu}$ while its rotational part to a combination of the $\sigma^{\mu \alpha}{ }_{\beta}$ 's.

3. For any vector field $\mathbf{K}$ one can form the vector field (Noether current)

$$
\begin{aligned}
C^{\mu}[\mathbf{K}]: & =\theta^{\mu}{ }_{\nu} K^{\nu}+\left(\sigma^{\mu[\alpha \beta]}+\sigma^{\alpha[\beta \mu]}+\sigma^{\beta[\alpha \mu]}\right) \nabla_{\alpha} K_{\beta}= \\
& =T^{\mu \nu} K_{\nu}+\nabla_{\nu}\left(\left(\sigma^{\mu[\nu \rho]}-\sigma^{\nu[\mu \rho]}-\sigma^{\rho[\mu \nu]}\right) K_{\rho}\right) .
\end{aligned}
$$

The difference $C^{\mu}[\mathbf{K}]-T^{\mu \nu} K_{\nu}$, being the divergence of an antisymmetric tensor, is identically conserved. Since $\nabla_{\mu} C^{\mu}[\mathbf{K}]=\nabla_{\mu}\left(T^{\mu \nu} K_{\nu}\right)=\frac{1}{2} T^{\mu \nu} \mathrm{E}_{\mathbf{K}} g_{\mu \nu}$, the vector fields $C^{\mu}[\mathbf{K}]$ and $T^{\mu \nu} K_{\nu}$ themselves are conserved if $\mathbf{K}$ is a Killing vector. The interpretation of the conserved vector fields $C^{\mu}[\mathbf{K}]$ depends not only on the Killing vector $\mathbf{K}$, or possibly on the global properties of its integral curves, but on the structure of the whole Lie algebra of the Killing vectors too. There is an obvious and commonly accepted interpretation of these conserved quantities only in the case of flat (Lorentz) geometry, yielding the interpretation of $\theta^{\mu}{ }_{\nu}$ as the canonical energy-momentum and $\sigma^{\mu \alpha}{ }_{\beta}$ as the canonical spin tensors of the matter fields: The Lie algebra of the Killing vectors is isomorphic to the semi direct sum of $\mathbf{R}^{m}$ and $s o(1, m-1)$, and hence the classification of the Killing vectors as "translations" or "boost-rotations" comes from the structure of the Lie algebra itself. In a Descartes coordinate system, adapted to the translation Killing vectors, the conserved vector field associated to the "translation" Killing vector $K_{\alpha}^{\mu}=\delta_{\alpha}^{\mu}$ is the linear momentum $C^{\mu}\left[\mathbf{K}_{\alpha}\right]=\theta^{\mu}{ }_{\alpha}$, and to the "rotation-boost" Killing vector $K_{\alpha \beta}^{\mu}=x^{\nu} g_{\nu \alpha} \delta_{\beta}^{\mu}-x^{\nu} g_{\nu \beta} \delta_{\alpha}^{\mu}$ is the angular momentum $C^{\mu}\left[\mathbf{K}_{\alpha \beta}\right]=g_{\alpha \nu} g_{\beta \rho}\left(\theta^{\mu \rho} x^{\nu}-\theta^{\mu \nu} x^{\rho}-2 \sigma^{\mu[\rho \nu]}\right)$. The last is the sum of the orbital momentum of $\theta^{\mu}{ }_{\nu}$ and some "internal" quantity: the sum of the orbital and spin angular momenta. If $\mathbf{K}$ is only a conformal Killing vector then the additional property $0=T^{\alpha}{ }_{\alpha}=\theta^{\alpha}{ }_{\alpha}+2 g_{\alpha \beta} \nabla_{\mu} \sigma^{\alpha[\beta \mu]}$ ensures the conservation of $C^{\mu}[\mathbf{K}]$ and $T^{\mu \nu} K_{\nu}[16]$.

Now consider the invariance properties of the quantities introduced so far under certain transformations of the Lagrangian and the fields.

a. Let $l^{\mu}$ be any vector field built up from $g_{\alpha \beta}$, the fields and possibly their first covariant derivatives; i.e. $l^{\mu}=l^{\mu}\left(g_{\alpha \beta}, \Phi^{n}, \nabla_{\beta} \Phi^{n}\right)$. Then the transformation $L \mapsto L+\nabla_{\mu} l^{\mu}$ leaves invariant 
both the Euler-Lagrange expressions, i.e. $E_{n}\left[L+\nabla_{\mu} l^{\mu}, \Phi\right]=E_{n}[L, \Phi]$, and the dynamical energymomentum tensor $T^{\alpha \beta}$, since they are functional derivatives of $I_{D}[g, \Phi]$. However, both $\theta^{\mu}{ }_{\nu}$ and $\sigma^{\mu \alpha}{ }_{\beta}$ will in general change: Since

$$
\frac{\partial}{\partial \nabla_{\mu} \Phi} \nabla_{\rho} l^{\rho}=\nabla_{\rho}\left(\frac{\partial l^{\rho}}{\partial \nabla_{\mu} \Phi}\right)+\frac{\partial l^{\mu}}{\partial \Phi}
$$

and

$$
\frac{\partial}{\partial \nabla_{\mu} \nabla_{\nu} \Phi} \nabla_{\rho} l^{\rho}=\frac{1}{2}\left(\frac{\partial l^{\mu}}{\partial \nabla_{\nu} \Phi}+\frac{\partial l^{\nu}}{\partial \nabla_{\mu} \Phi}\right)
$$

one has

$$
\begin{aligned}
\sigma_{\beta}^{\mu \alpha} \mapsto \sigma_{\beta}^{\mu \alpha} & +\frac{\partial l^{\mu}}{\partial \Phi^{n}} \Delta_{\beta}^{\alpha} \Phi^{n}+\frac{\partial l^{\mu}}{\partial \nabla_{\rho} \Phi^{n}} \Delta_{\beta}^{\alpha} \nabla_{\rho} \Phi^{n}- \\
& -\frac{1}{2}\left(\frac{\partial l^{\mu}}{\partial \nabla_{\alpha} \Phi^{n}}+\frac{\partial l^{\alpha}}{\partial \nabla_{\mu} \Phi^{n}}\right) \nabla_{\beta} \Phi^{n}+ \\
& +\nabla_{\rho}\left(\frac{1}{2}\left[\frac{\partial l^{\rho}}{\partial \nabla_{\mu} \Phi^{n}}-\frac{\partial l^{\mu}}{\partial \nabla_{\rho} \Phi^{n}}\right] \Delta_{\beta}^{\alpha} \Phi^{n}\right)
\end{aligned}
$$

and

$$
\begin{aligned}
\theta_{\nu}^{\mu} \mapsto \theta_{\nu}^{\mu} & +\nabla_{\rho} l^{\rho} \delta_{\nu}^{\mu}-\frac{\partial l^{\mu}}{\partial \Phi^{n}} \nabla_{\nu} \Phi^{n}-\frac{\partial l^{\mu}}{\partial \nabla_{\rho} \Phi^{n}} \nabla_{\nu} \nabla_{\rho} \Phi^{n}- \\
& -\frac{1}{2} \nabla_{\rho}\left(\frac{\partial l^{\rho}}{\partial \nabla_{\mu} \Phi^{n}}-\frac{\partial l_{\mu}}{\partial \nabla_{\rho} \Phi^{n}}\right) \nabla_{\nu} \Phi^{n}+ \\
& -\frac{1}{2}\left(\frac{\partial l^{\rho}}{\partial \nabla_{\mu} \Phi^{n}}-\frac{\partial l^{\mu}}{\partial \nabla_{\rho} \Phi^{n}}\right) \nabla_{\nu} \nabla_{\rho} \Phi^{n} .
\end{aligned}
$$

Similarly, $T^{\alpha \beta} K_{\beta}$ does not change if the Lagrangian is modified by a total divergence, but in general $C^{\mu}[\mathbf{K}]$ does.

b. Suppose that the matter fields have some internal symmetry, characterized by a Lie group $G$ and a linear representation of it on some $N$ dimensional vector space $V$; and the matter fields are either particle or gauge (connection) fields. The action of $G$ on $V$ mixes linearly the particle fields $\Psi_{\beta_{1} \ldots \beta_{s}}^{P \alpha_{1} \ldots \alpha_{r}}, P=1, \ldots, N$, while the remaining matter fields, the connection fields, are $\mathcal{G}$-valued 1 forms $A_{\mu}^{\Gamma}$ ( $\mathcal{G}$ is the Lie algebra of $G$ ), and they transform according to the adjoint representation of $G$. Let $\left\{\lambda_{\Gamma Q}^{P}\right\}$ be the basis of a linear representation of $\mathcal{G}$ on $V, \Gamma=1, \ldots, \operatorname{dim} G$, and $c_{\Delta \Pi}^{\Gamma}$ the structure constants. Then any gauge invariant matter Lagrangian with minimal coupling must have the form [17]: $L_{m}=L_{p}\left(g_{\alpha \beta}, \Psi^{P}, D_{\mu} \Psi^{P}, D_{\mu} D_{\nu} \Psi^{P}\right)+L_{c}\left(g_{\alpha \beta}, F^{P}{ }_{Q \mu \nu}\right)$, where $D_{\mu} \Psi^{P}:=\nabla_{\mu} \Psi^{P}+$ $A_{\mu}^{\Gamma} \lambda_{\Gamma Q}^{P} \Psi^{Q}$ is the spacetime-gauge covariant derivative and $F_{\mu \nu}^{\Gamma}:=\partial_{\mu} A_{\nu}^{\Gamma}-\partial_{\nu} A_{\mu}^{\Gamma}+c_{\Delta \Pi}^{\Gamma} A_{\mu}^{\Delta} A_{\nu}^{\Pi}$ is the field strength of the connection fields. If $L_{m}$ is gauge invariant, or it can be made gauge invariant by adding to it a total divergence $\nabla_{\mu} l^{\mu}$ then the dynamical energy momentum tensor $T^{\alpha \beta}$ is gauge invariant too. But in general neither $\theta^{\mu}{ }_{\nu}$ nor $\sigma^{\mu \alpha}{ }_{\beta}$ is gauge invariant. $T^{\alpha \beta} K_{\alpha}$ is gauge invariant also, but in general $C^{\mu}[\mathbf{K}]$ is not.

If the spacetime geometry is specified by a (local) field of basis 1 forms $\left\{\vartheta_{\alpha}^{a}\right\}$ and a constant matrix $\eta_{a b}$ for which $\eta_{a b}=\eta_{e f} \Lambda_{a}^{e} \Lambda^{f}{ }_{b} \forall \Lambda^{i}{ }_{j} \in O(p, q)$ then $g_{\alpha \beta}:=\vartheta_{\alpha}^{a} \vartheta_{\beta}^{b} \eta_{a b}$ is a metric of $p-q$ signature and the analysis above can be repeated. The requirement of the local $O(p, q)$-invariance of the matter Lagrangian implies $L=L_{m}\left(\Phi_{b_{1} \ldots b_{s}}^{n a_{1} \ldots a_{r}}, \nabla_{e} \Phi_{b_{1} \ldots b_{s}}^{n a_{1} \ldots a_{r}}, \nabla_{e} \nabla_{f} \Phi_{b_{1} \ldots b_{s}}^{n a_{1} \ldots a_{r}}\right)$ and $\theta^{[a b]}=-\nabla_{e} \sigma^{e[a b]}$, 
where e.g. $\quad \nabla_{e} \Phi_{b_{1} \ldots b_{s}}^{n a_{1} \ldots a_{r}}:=\nabla_{\mu} \Phi_{\beta_{1} \ldots \beta_{s}}^{n \alpha_{1} \ldots \alpha_{r}} \vartheta_{\alpha_{1}}^{a_{1}} \ldots \vartheta_{\alpha_{r}}^{a_{r}} E_{e}^{\mu} E_{b_{1}}^{\beta_{1}} \ldots E_{b_{s}}^{\beta_{s}}$. Thus the algebraic BelinfanteRosenfeld equation is a consequence of the $O(p, q)$-invariance of $L_{m}$, independently of the field equations, and hence the Belinfante-Rosenfeld combination of $\theta^{\mu}{ }_{\nu}$ and $\sigma^{\mu \alpha}{ }_{\beta}$ is symmetric. The functional derivative $T^{\alpha}{ }_{a}$ of $I_{D}[\vartheta, \Phi]$ with respect to $\vartheta_{\alpha}^{a}$ is $E_{e}^{\alpha} \eta_{a f}\left(\theta^{[e f]}+\nabla_{k}\left(\sigma^{k[e f]}+\sigma^{e[f k]}+\sigma^{f[e k]}\right)\right)$ $+E_{e}^{\alpha} E_{n}[L, \Phi] \Delta_{a}^{e} \Phi^{n}$, and hence the matter field equations imply the symmetry of $T^{a b}:=\vartheta_{\alpha}^{a} T^{\alpha}{ }_{e} \eta^{e b}$. Moreover, since $\nabla_{a} T^{a b}=\nabla_{a} \theta^{a b}+R^{b}{ }_{i j k} \sigma^{i j k}=E_{n}[L, \Phi] \eta^{b c} \nabla_{c} \Phi^{n}$, the field equations imply the vanishing of $\nabla_{a} T^{a b}$; and hence the differential Belinfante-Rosenfeld equation.

\subsection{Canonical pseudotensors of gravity}

The formalism described in the previous section cannot be applied to gravity in its original form, since a sharp distinction between the field variables and the fields specifying the spacetime geometry was needed, while for gravity these two coincide. The gravitational action is therefore built only from the gravitational field variables and thus one can form only its functional derivative with respect to the gravitational field variables. These are the Euler-Lagrange expressions but we do not have any gravitational counterpart of the dynamical energy momentum tensor. Thus if we want to describe the energy-momentum (and possibly angular momentum) content of gravity the gravitational counterpart of the canonical energy-momentum and spin tensors should be introduced. But since

$$
E^{\alpha \beta}[R, g] \mathrm{E}_{\mathbf{K}} g_{\alpha \beta}=-\left(R^{\alpha \beta}-\frac{1}{2} R g^{\alpha \beta}\right) \mathrm{Ł}_{\mathbf{K}} g_{\alpha \beta}=-2 \nabla_{\alpha}\left(\left(R_{\beta}^{\alpha}-\frac{1}{2} R \delta_{\beta}^{\alpha}\right) K^{\beta}\right)
$$

thus, at least for Hilbert's scalar Lagrangian $\frac{1}{2 \kappa} R$ of Einstein's theory, the comparison of the equation above with (2.1.10.) shows that the canonical energy momentum tensor of gravity would be $-\frac{1}{\kappa} G_{\beta}^{\alpha}$ while the canonical spin tensor would be zero. In the weak field approximation, however, gravity behaves as a spin two field on Minkowski's spacetime; and hence in general non-vanishing spin tensor and non-symmetric canonical energy-momentum tensor would be expected. (If the first Noether theorem were used for Hilbert's gravitational action then in the big round brackets on the right hand side Komar's expression $\frac{1}{2} \nabla_{\beta}\left(\nabla^{\beta} K^{\alpha}-\nabla^{\alpha} K^{\beta}\right)$ would also appear [6]. However Komar's expression is identically conserved and contains the second derivative of $\mathbf{K}$, and hence it cannot be considered as the spin term.)

A formalism analogous to that described in the previous paragraph can however be developed by choosing a local coordinate system and using partial derivations as torsion free flat covariant derivations [10]. This coordinate system defines a local background geometry on which the physical metric becomes a field, and hence the scenario of the Lagrangian field theory can be applied to it. (Another approach is to choose a non-dynamical background metric [18] or connection [19] on $M$, and to "covariantize" the formalism. Although the limitation of the first, coordinate approach is obvious: the results are valid only in a coordinate neighbourhood, we choose this approach since here we are interested only in local expressions, and locally all three approaches are essentially equivalent.) This yields the so-called canonical energy-momentum and spin pseudotensors (or complexes) of gravity, according to the following formal prescription:

Choose a local coordinate neighbourhood $U \subseteq M$ with coordinates $\left(x^{1}, \ldots, x^{m}\right)$ on $U$ and the collection $\phi^{n}$ of gravitational field variables. $\phi^{n}$ may be, for example, the metric tensor $g_{\alpha \beta}$ or a collection $\left\{\vartheta_{\alpha}^{a}\right\}$ of basis 1 forms satisfying $g^{\alpha \beta} \vartheta_{\alpha}^{a} \vartheta_{\beta}^{b}=\eta^{a b}$ for some constant matrix 
$\eta^{a b}, a, b=1, \ldots, m$. The first description will be called holonomic while the second anholonomic. The gravitational Lagrangian $\mathcal{L}$ will be assumed not to depend on higher than the second derivatives of $\phi^{n}: \mathcal{L}=\mathcal{L}\left(\phi^{n}, \partial_{\mu} \phi^{n}, \partial_{\mu} \partial_{\nu} \phi^{n}\right)$ and the gravitational action functional is $I_{D}[\phi]:=\int_{D} \mathcal{L}\left(\phi^{n}(x), \partial_{\mu} \phi^{n}(x), \partial_{\mu} \partial_{\nu} \phi^{n}(x)\right) d^{m} x, D \subseteq M$. If $g$ is the determinant of $g_{\alpha \beta}$ in the coordinate system $\left(x^{1}, \ldots, x^{m}\right)$ then the canonical energy-momentum and spin pseudotensors, $t^{\mu}{ }_{\nu}$ and $s^{\mu \alpha}$, are defined by

$$
\begin{aligned}
\sqrt{|g|} t^{\mu}{ }_{\nu}:=\mathcal{L} \delta_{\nu}^{\mu} & -\left(\frac{\partial \mathcal{L}}{\partial \partial_{\mu} \phi^{n}}-\partial_{\rho}\left(\frac{\partial \mathcal{L}}{\partial \partial_{\mu} \partial_{\rho} \phi^{n}}\right)\right) \partial_{\nu} \phi^{n}- \\
& -\frac{\partial \mathcal{L}}{\partial \partial_{\mu} \partial_{\rho} \phi^{n}} \partial_{\nu} \partial_{\rho} \phi^{n}
\end{aligned}
$$

and

$$
\begin{aligned}
\sqrt{|g|} s^{\mu \alpha}{ }_{\beta}: & =\left(\frac{\partial \mathcal{L}}{\partial \partial_{\mu} \phi^{n}}-\partial_{\rho}\left(\frac{\partial \mathcal{L}}{\partial \partial_{\mu} \partial_{\rho} \phi^{n}}\right)\right) \Delta_{\beta}^{\alpha} \phi^{n}+ \\
& +\frac{\partial \mathcal{L}}{\partial \partial_{\mu} \partial_{\rho} \phi^{n}}\left(\partial_{\rho} \Delta_{\beta}^{\alpha} \phi^{n}-\delta_{\rho}^{\alpha} \partial_{\beta} \phi^{n}\right)
\end{aligned}
$$

respectively. Assuming the gravitational field equations to hold, $\mathcal{E}_{n}\left[\mathcal{L}+\mathcal{L}_{m}, \phi\right]:=\frac{\partial\left(\mathcal{L}+\mathcal{L}_{m}\right)}{\partial \phi^{n}}-$ $\partial_{\mu}\left(\frac{\partial \mathcal{L}+\mathcal{L}_{m}}{\partial \partial_{\mu} \phi^{n}}\right)+\partial_{\mu} \partial_{\nu}\left(\frac{\partial \mathcal{L}+\mathcal{L}_{m}}{\partial \partial_{\mu} \partial_{\nu} \phi^{n}}\right)=0$, where $\mathcal{L}_{m}:=\sqrt{|g|} L_{m}$ is considered as a function of the gravitational and matter field variables and their first and possibly the second partial derivatives, the coordinate (or pseudo) divergence of (2.2.2.)

$$
\begin{aligned}
\partial_{\mu}\left(\sqrt{|g|} t^{\mu}{ }_{\nu}\right) & =\mathcal{E}_{n}[\mathcal{L}, \phi] \partial_{\nu} \phi^{n}= \\
& =-\mathcal{E}_{n}\left[\mathcal{L}_{m}, \phi\right] \partial_{\nu} \phi^{n} .
\end{aligned}
$$

But the functional derivative $\mathcal{E}_{n}\left[\mathcal{L}_{m}, \phi\right]$ is essentially the dynamical energy-momentum tensor of the matter fields. Thus, assuming the matter field equations to hold, one finally has

$$
\partial_{\mu}\left(\sqrt{|g|}\left(t^{\mu}{ }_{\nu}+T^{\mu}{ }_{\nu}\right)\right)=0
$$

This equation implies the existence of a so-called superpotential $\cup_{\nu}^{\mu \alpha}=\cup_{\nu}{ }^{[\mu \alpha]}$ such that

$$
\sqrt{|g|}\left(t^{\mu}{ }_{\nu}+T^{\mu}{ }_{\nu}\right)=\frac{1}{2} \partial_{\alpha} \cup_{\nu}{ }^{\mu \alpha} .
$$

In the theory of pseudotensors of general relativity $[4-10,18,20,21,25,26]$ one usually starts with a more or less ad hoc superpotential, built from the gravitational field variables, and using Einstein's equations $\kappa T^{\mu}{ }_{\nu}=G^{\mu}{ }_{\nu}:=R^{\mu}{ }_{\nu}-\frac{1}{2} R \delta_{\nu}^{\mu}$ one defines the energy-momentum pseudotensor by eq.(2.2.6.). But now one has as many pseudotensors as the equivalence classes of $\cup_{\beta}{ }^{\alpha \mu}$ 's, where $\cup$ and $\tilde{U}$ are equivalent if $\cup_{\beta}{ }^{\alpha \mu}-\tilde{\cup}_{\beta}{ }^{\alpha \mu}=\partial_{\nu} V_{\beta}{ }^{\alpha \mu \nu}$ for some $V_{\beta}{ }^{\alpha \mu \nu}=V_{\beta}{ }^{\alpha[\mu \nu]}=V_{\beta}{ }^{[\alpha \mu] \nu}$. Thus in the sea of pseudotensors it is rather difficult to find which pseudotensors may have physical significance (if any). On the other hand the Lagrangian formalism above may be considered as a selection rule to choose from the mathematically possible pseudotensors. This is the reason why we prefer this approach of pseudotensors and consider only the so-called canonical ones.

If the gravitational Lagrangian is modified as $\mathcal{L} \mapsto \mathcal{L}+\partial_{\mu} l^{\mu}$ for some $l^{\mu}=l^{\mu}\left(\phi^{n}, \partial_{\rho} \phi^{n}\right)$, then $t^{\mu}{ }_{\nu}$ and $s^{\mu \alpha}{ }_{\beta}$ will in general change according to 


$$
\begin{aligned}
\sqrt{|g|} t^{\mu}{ }_{\nu} \mapsto \sqrt{|g|} t^{\mu}{ }_{\nu} & +\partial_{\rho} l^{\rho} \delta_{\nu}^{\mu}-\frac{\partial l^{\mu}}{\partial \phi^{n}} \partial_{\nu} \phi^{n}-\frac{\partial l^{\mu}}{\partial \partial_{\rho} \phi^{n}} \partial_{\nu} \partial_{\rho} \phi^{n}- \\
& -\frac{1}{2} \partial_{\rho}\left(\left[\frac{\partial l^{\rho}}{\partial \partial_{\mu} \phi^{n}}-\frac{\partial l^{\mu}}{\partial \partial_{\rho} \phi^{n}}\right] \partial_{\nu} \phi^{n}\right)
\end{aligned}
$$

and

$$
\begin{aligned}
\sqrt{|g|} s_{\beta}^{\mu \alpha} \mapsto \sqrt{|g|} s_{\beta}^{\mu \alpha} & +\frac{\partial l^{\mu}}{\partial \phi^{n}} \Delta_{\beta}^{\alpha} \phi^{n}+\frac{\partial l^{\mu}}{\partial \partial_{\rho} \phi^{n}} \Delta_{\beta}^{\alpha} \partial_{\rho} \phi^{n} \\
& -\frac{1}{2}\left(\frac{\partial l^{\mu}}{\partial \partial_{\alpha} \phi^{n}}+\frac{\partial l^{\alpha}}{\partial \partial_{\mu} \phi^{n}}\right) \partial_{\beta} \phi^{n}+ \\
& +\frac{1}{2} \partial_{\rho}\left(\left[\frac{\partial l^{\rho}}{\partial \partial_{\mu} \phi^{n}}-\frac{\partial l^{\mu}}{\partial \partial_{\rho} \phi^{n}}\right] \Delta_{\beta}^{\alpha} \phi^{n}\right) .
\end{aligned}
$$

The price we have to pay for this construction is the non tensorial character of these quantities, they are highly coordinate dependent. Because of the noncommutativity of partial derivations with index risings and lowering, it will not always be trivial which quantities will be useful. For example an equation may be extremely complicated if covariant indexes are used, while remarkably simple in its contravariant form.

In the next paragraphs the canonical pseudotensors of Einstein's theory, based first on Einstein's first order and then Hilbert's second order Lagrangian, will be studied both in holonomic and anholonomic descriptions.

\subsection{Einstein's pseudotensors in holonomic description}

The gravitational field variables are the components $g_{\alpha \beta}$ of the metric in a fixed coordinate system $\left(x^{1}, \ldots, x^{m}\right)$ and Einstein's Lagrangian is

$$
\begin{aligned}
\mathcal{L}_{E}: & =\frac{1}{2 \kappa}\left\{R \sqrt{|g|}-\partial_{\mu}\left(\sqrt{|g|}\left(\Gamma_{\gamma \delta}^{\mu} g^{\gamma \delta}-g^{\mu \rho} \Gamma_{\rho \nu}^{\nu}\right)\right)\right\}= \\
& =\frac{1}{2 \kappa} \sqrt{|g|} g^{\gamma \delta}\left(\Gamma_{\gamma \nu}^{\rho} \Gamma_{\delta \rho}^{\nu}-\Gamma_{\rho \nu}^{\rho} \Gamma_{\gamma \delta}^{\nu}\right),
\end{aligned}
$$

where $\kappa>0$ is Einstein's gravitational constant. For later convenience let us define

$$
l^{\mu}:=\sqrt{|g|}\left(\Gamma_{\gamma \delta}^{\mu} g^{\gamma \delta}-g^{\mu \rho} \Gamma_{\rho \nu}^{\nu}\right)=\sqrt{|g|} g^{\mu \nu}\left(\partial_{\alpha} g_{\nu \beta}-\partial_{\nu} g_{\alpha \beta}\right) g^{\alpha \beta} .
$$

Thus $2 \kappa \mathcal{L}_{E}=R \sqrt{|g|}-\partial_{\mu} l^{\mu}$. Einstein's energy-momentum pseudotensor [10] is defined by (2.2.2.) and (2.3.1.):

$$
\begin{aligned}
2 \kappa \sqrt{|g|_{E}} t_{\beta}^{\alpha}: & =2 \kappa\left(\mathcal{L}_{E} \delta_{\beta}^{\alpha}-\frac{\partial \mathcal{L}_{E}}{\partial \partial_{\alpha} g_{\mu \nu}} \partial_{\beta} g_{\mu \nu}\right)= \\
& =\sqrt{|g|}\left\{\delta_{\beta}^{\alpha}\left(\Gamma_{\nu \gamma}^{\rho} \Gamma_{\rho \delta}^{\nu}-\Gamma_{\rho \nu}^{\rho} \Gamma_{\gamma \delta}^{\nu}\right) g^{\gamma \delta}+g^{\alpha \rho} \Gamma_{\rho \beta}^{\nu} \Gamma_{\gamma \nu}^{\gamma}-\right. \\
& \left.-g^{\alpha \rho} \Gamma_{\rho \nu}^{\nu} \Gamma_{\beta \mu}^{\mu}+\Gamma_{\gamma \delta}^{\alpha} g^{\gamma \delta} \Gamma_{\beta \nu}^{\nu}+\Gamma_{\beta \gamma}^{\alpha} \Gamma_{\rho \delta}^{\rho} g^{\gamma \delta}-2 \Gamma_{\rho \gamma}^{\alpha} \Gamma_{\beta \delta}^{\rho} g^{\gamma \delta}\right\} .
\end{aligned}
$$

${ }_{E} t^{\alpha}{ }_{\beta}$ can be recovered from P. von Freud's superpotential [4-8,10]: If $H^{\alpha \beta \gamma \delta}:=|g| G^{\alpha \beta \gamma \delta}:=$ $|g|\left(g^{\alpha \gamma} g^{\beta \delta}-g^{\alpha \delta} g^{\beta \gamma}\right)$ - which is called the Landau-Lifshitz superpotential - then von Freud's superpotential is defined by 


$$
\begin{aligned}
{ }_{F} \cup_{\rho}{ }^{\alpha \beta}: & =\frac{1}{\sqrt{|g|}} g_{\rho \gamma} \partial_{\delta} H^{\alpha \beta \gamma \delta}= \\
& =-2 \sqrt{|g|}\left(\Gamma_{\rho \mu}^{[\alpha} g^{\beta] \mu}+\delta_{\rho}^{[\alpha} \Gamma_{\gamma \delta}^{\beta]} g^{\gamma \delta}-\delta_{\rho}^{[\alpha} g^{\beta] \mu} \Gamma_{\nu \mu}^{\nu}\right) .
\end{aligned}
$$

Trivially ${ }_{F} \cup{ }_{\rho}{ }^{\alpha \beta}={ }_{F} \cup_{\rho}{ }^{[\alpha \beta]},{ }_{F} \cup^{[\rho \alpha \beta]}=0$ and ${ }_{F} \cup_{\rho}{ }^{\alpha \rho}=(m-2) l^{\mu}$. Now the so-called von Freud equation is

$$
2 \sqrt{|g|}\left(G^{\alpha}{ }_{\beta}+\kappa_{E} t^{\alpha}{ }_{\beta}\right)=\partial_{\mu F} \cup_{\beta}{ }^{\alpha \mu} .
$$

Because of the antisymmetry of $F_{F} \cup_{\rho}^{\alpha \beta}$ (2.3.5.) implies the (pseudo)divergence equation $\partial_{\alpha}(\sqrt{|g|}$ $\left.\left(G^{\alpha}{ }_{\beta}+\kappa_{E} t^{\alpha}{ }_{\beta}\right)\right)=0$. Since $\partial_{\alpha} \partial_{\beta}=\partial_{\beta} \partial_{\alpha}$ this pseudo divergence equation looks like as the differential Belinfante-Rosenfeld equation. ${ }_{E} t^{\alpha}{ }_{\beta}$ does not determine the superpotential uniquely: ${ }_{F} \cup_{\beta}{ }^{\alpha \mu}$ and ${ }_{F} \cup_{\beta}{ }^{\alpha \mu}+\partial_{\nu} V_{\beta}{ }^{\alpha \mu \nu}$ belong to the same pseudotensor, ${ }_{E} t^{\alpha}{ }_{\beta}$, for any $V_{\beta}{ }^{\alpha \mu \nu}=V_{\beta}{ }^{\alpha[\mu \nu]}$ $=V_{\beta}{ }^{[\alpha \mu] \nu}$, which therefore has the symmetry $V_{\beta}{ }^{\alpha \mu \nu}=V_{\beta}{ }^{[\alpha \mu \nu]}$.

Using definition (2.2.3.) we introduce the canonical spin pseudotensor:

$$
\begin{aligned}
2 \kappa \sqrt{|g|}{ }_{E} s_{\beta}^{\mu \alpha}: & =2 \kappa \frac{\partial \mathcal{L}_{E}}{\partial \partial_{\mu} g_{\rho \nu}}\left(-\delta_{\rho}^{\alpha} g_{\nu \beta}-\delta_{\nu}^{\alpha} g_{\rho \beta}\right)= \\
& =\sqrt{|g|}\left\{\delta_{\beta}^{\alpha}\left(\Gamma_{\gamma \delta}^{\mu} g^{\gamma \delta}-g^{\mu \rho} \Gamma_{\rho \nu}^{\nu}\right)+g^{\mu \alpha} \Gamma_{\beta \rho}^{\rho}+\delta_{\beta}^{\mu} g^{\alpha \rho} \Gamma_{\rho \gamma}^{\gamma}-2 g^{\alpha \rho} \Gamma_{\rho \beta}^{\mu}\right\}
\end{aligned}
$$

This spin pseudotensor and von Freud's superpotential are not quite independent since their sum is a total coordinate divergence:

$$
\begin{aligned}
2 \kappa \sqrt{|g|}_{E} s^{\mu \alpha}{ }_{\beta}+{ }_{F} \cup_{\beta}{ }^{\alpha \mu} & =\frac{1}{2}\left(g^{\mu \alpha} \Gamma_{\rho \beta}^{\rho}-g^{\alpha \rho} \Gamma_{\rho \beta}^{\mu}-g^{\mu \rho} \Gamma_{\rho \beta}^{\alpha}+\delta_{\beta}^{\mu} \Gamma_{\gamma \delta}^{\alpha} g^{\gamma \delta}\right)= \\
& =\partial_{\nu}\left(\sqrt{|g|} G^{\alpha \rho \mu \nu} g_{\rho \beta}\right) .
\end{aligned}
$$

Thus although the canonical energy-momentum pseudotensor determines only a class of superpotentials and does not specify any element of this class, the canonical spin pseudotensor does single out uniquely a superpotential: Since for $V_{\beta}{ }^{\alpha \mu \nu}:=\sqrt{|g|} g_{\beta \rho} G^{\rho \alpha \mu \nu}$ one has $V_{\beta}{ }^{[\alpha \mu \nu]}=0$, thus the superpotential that the canonical spin pseudotensor specifies is just the von Freud superpotential. ${ }_{F} \cup_{\beta}{ }^{\alpha \mu}$ therefore has distinguished role among the superpotentials associated to ${ }_{E} t^{\alpha}{ }_{\beta}$. However, although for general $V_{\beta}{ }^{\alpha \mu \nu}=V_{\beta}{ }^{\alpha[\mu \nu]}$ the sum ${ }_{F} \cup_{\beta}{ }^{\alpha \mu}+\partial_{\nu} V_{\beta}{ }^{\alpha \mu \nu}$ is not antisymmetric in $\alpha$ and $\mu$, and hence it is not a superpotential in the strict sense, but its $\partial_{\mu}$-divergence is just the right hand side of (2.3.5.). Thus it can be considered as a superpotential in a more general sense. (The Landau-Lifshitz superpotential is not superpotential in either sense, but because of historical reasons we retain this terminology.) In this sense the canonical spin pseudotensor is a superpotential for the canonical energy-momentum pseudotensor:

$$
\sqrt{|g|}\left(G_{\beta}^{\alpha}+\kappa_{E} t^{\alpha}{ }_{\beta}\right)=-\partial_{\mu}\left(\kappa \sqrt{|g|}_{E} s^{\mu \alpha}{ }_{\beta}\right) .
$$

It is a remarkable property of (2.3.8.) that it contains only canonically defined quantities. In the classical Lagrangian theory of matter fields the canonical energy momentum tensor is not a source of the canonical spin tensor, one has only the algebraic Belinfante-Rosenfeld equation (2.1.12.). 
Thus the fact that the (pseudo)source of the canonical spin pseudotensor is the canonical energy momentum pseudotensor seems to be a characteristic feature of gravity. Although the definition (2.3.6.) and the equations (2.3.7.), (2.3.8.) appeared in the literature $[20,22]$ but it was not identified as the canonical spin pseudotensor of gravity, ${ }_{E} s^{\mu \alpha}{ }_{\beta}$ was introduced only as a useful auxiliary quantity.

Recalling the noncommutativity of partial derivation and index rising, the antisymmetric part of (2.3.8.),

$$
\sqrt{|g|}\left({ }_{E} t^{\alpha \beta}-{ }_{E} t^{\beta \alpha}\right)=-\partial_{\mu}\left(\sqrt{|g|}_{E} s_{\rho}^{\mu \alpha}\right) g^{\rho \beta}+\partial_{\mu}\left(\sqrt{|g|}_{E} s_{\rho}^{\mu \beta}\right) g^{\rho \alpha},
$$

takes just the form of the algebraic Belinfante-Rosenfeld equation (2.1.12.). To transform (2.3.9.) to the usual form of the algebraic Belinfante-Rosenfeld equation, the contravariant form of the energy-momentum and spin pseudotensors are needed.

First consider the contravariant form of ${ }_{E} t^{\alpha}{ }_{\beta}$. In general ${ }_{E} t^{\alpha \beta}:={ }_{E} t^{\alpha}{ }_{\rho} g^{\rho \beta}$ is not symmetric, and, since partial derivation and index rising do not commute, $2 \sqrt{|g|}\left(G^{\alpha \beta}+\kappa_{E} t^{\alpha \beta}\right)$ is not $\partial_{\alpha^{-}}$ divergence free. But from von Freud's equation we have

$$
2 \sqrt{|g|}\left(G^{\alpha \beta}+\kappa_{E} t^{\alpha \beta}\right)=\partial_{\mu}\left(g_{F}^{\beta \rho} \cup_{\rho}^{\alpha \mu}\right)-\partial_{\mu} g_{F}^{\beta \rho} \cup_{\rho}^{\alpha \mu} .
$$

Therefore if we define

$$
\begin{aligned}
\kappa_{E} \theta^{\alpha \beta}: & =\kappa_{E} t^{\alpha \beta}+\frac{1}{2 \sqrt{|g|}} \partial_{\mu} g_{F}^{\beta \rho} \cup_{\rho}^{\alpha \mu}= \\
& =\frac{1}{2}\left\{g^{\alpha \beta}\left(\Gamma_{\nu \gamma}^{\rho} \Gamma_{\rho \delta}^{\nu}-\Gamma_{\rho \nu}^{\rho} \Gamma_{\gamma \delta}^{\nu}\right) g^{\gamma \delta}+g^{\alpha \gamma} g^{\beta \delta} \Gamma_{\nu \gamma}^{\rho} \Gamma_{\rho \delta}^{\nu}+\Gamma_{\rho \gamma}^{\alpha} \Gamma_{\nu \delta}^{\beta} g^{\rho \nu} g^{\gamma \delta}-\right. \\
& -\Gamma_{\gamma \delta}^{\alpha} g^{\gamma \delta} \Gamma_{\rho \nu}^{\beta} g^{\rho \nu}-g^{\alpha \gamma} g^{\beta \delta} \Gamma_{\rho \gamma}^{\nu} \Gamma_{\nu \delta}^{\rho}-g^{\alpha \rho} \Gamma_{\rho \gamma}^{\nu} \Gamma_{\nu \delta}^{\beta} g^{\gamma \delta}-g^{\beta \rho} \Gamma_{\rho \gamma}^{\nu} \Gamma_{\nu \delta}^{\alpha} g^{\gamma \delta}- \\
& \left.+g^{\alpha \rho} \Gamma_{\rho \nu}^{\beta} \Gamma_{\gamma \delta}^{\nu} g^{\gamma \delta}+g^{\beta \rho} \Gamma_{\rho \nu}^{\alpha} \Gamma_{\gamma \delta}^{\nu} g^{\gamma \delta}+g^{\alpha \rho} \Gamma_{\rho \nu}^{\nu} \Gamma_{\gamma \delta}^{\beta} g^{\gamma \delta}-g^{\alpha \rho} \Gamma_{\rho \gamma}^{\beta} \Gamma_{\nu \delta}^{\nu} g^{\gamma \delta}\right\}
\end{aligned}
$$

then we obtain

$$
2 \sqrt{|g|}\left(G^{\alpha \beta}+\kappa_{E} \theta^{\alpha \beta}\right)=\partial_{\mu}\left(g_{F}^{\beta \rho} \cup_{\rho}^{\alpha \mu}\right) .
$$

The pseudotensor ${ }_{E} \theta^{\alpha \beta}$ is therefore associated to the contravariant form of von Freud's superpotential, and hence we have the (pseudo)divergence equation $\partial_{\alpha}\left(\sqrt{|g|}\left(G^{\alpha \beta}+\kappa_{E} \theta^{\alpha \beta}\right)\right)=0$. Therefore we would have to consider ${ }_{E} \theta^{\alpha \beta}$ as the "true" contravariant form of Einstein's canonical energymomentum pseudotensor instead of ${ }_{E} t^{\alpha \beta}$. This is just the pseudotensor known as Bergmann's energy-momentum pseudotensor $[18,23] .{ }_{E} \theta^{\alpha \beta}$ is a quadratic expression of the Christoffel symbols and contains only the first partial derivatives of the metric.

${ }_{E} \theta^{\alpha \beta}$ is not symmetric either, because

$$
4 \kappa_{E} \theta^{[\alpha \beta]}=g^{\gamma \delta}\left(g^{\alpha \rho} \Gamma_{\rho \nu}^{\nu} \Gamma_{\gamma \delta}^{\beta}-g^{\beta \rho} \Gamma_{\rho \nu}^{\nu} \Gamma_{\gamma \delta}^{\alpha}-g^{\alpha \rho} \Gamma_{\rho \gamma}^{\beta} \Gamma_{\nu \delta}^{\nu}+g^{\beta \rho} \Gamma_{\rho \gamma}^{\alpha} \Gamma_{\nu \delta}^{\nu}\right)
$$

Taking the antisymmetric part of (2.3.11.) and using ${ }_{F} \cup^{[\rho \alpha \mu]}=0$ we obtain an equation for ${ }_{E} \theta^{[\alpha \beta]}$ :

$$
\begin{aligned}
4 \kappa \sqrt{|g|}_{E} \theta^{[\alpha \beta]} & =\partial_{\mu}\left(g^{\beta \rho}{ }_{F} \cup_{\rho}{ }^{\alpha \mu}-g^{\alpha \rho}{ }_{F} \cup_{\rho}^{\beta \mu}\right)= \\
& =\partial_{\mu}\left(g^{\mu \rho}{ }_{F} \cup_{\rho}{ }^{\alpha \beta}\right)
\end{aligned}
$$


If we defined $2 \kappa \sqrt{|g|}_{E} \sigma^{\mu[\alpha \beta]}:=-\frac{1}{2} g^{\mu \rho}{ }_{F} \cup_{\rho}^{\alpha \beta}$ then (2.3.12.) would take the form

$$
\sqrt{|g|}_{E} \theta^{[\alpha \beta]}=-\partial_{\mu}\left(\sqrt{|g|}_{E} \sigma^{\mu[\alpha \beta]}\right)
$$

which looks like as the algebraic Belinfante-Rosenfeld equation (2.1.12.). (The analogy with (2.1.12.) could be made closer observing that $4 \kappa_{E} \theta^{[\alpha \beta]}=\partial_{\mu}\left(\frac{1}{\sqrt{|g|}} \partial_{\nu}\left(\sqrt{|g|} G^{\alpha \beta \mu \nu}\right)\right)$, since with $4 \kappa \sqrt{|g|} \tilde{\sigma}^{\mu[\alpha \beta]}:=-\partial_{\nu}\left(\sqrt{|g|} G^{\alpha \beta \mu \nu}\right)$ we would have ${ }_{E} \theta^{[\alpha \beta]}=-\partial_{\mu E} \tilde{\sigma}^{\mu[\alpha \beta]}$. However the coefficient $\sqrt{|g|}$ appears now naturally and the structure of the equations suggest to retain those forms of equations that contain $\sqrt{|g|}$.)

For the contravariant form of the spin pseudotensor we define

$$
\begin{aligned}
2 \kappa \sqrt{|g|}_{E} \sigma^{\mu \alpha \beta}: & =2 \kappa \sqrt{|g|}_{E} s^{\mu \alpha \beta}+\sqrt{|g|} G^{\alpha \gamma \mu \nu} g_{\gamma \rho} \partial_{\nu} g^{\rho \beta}-\partial_{\nu}\left(\sqrt{|g|} G^{\alpha \beta \mu \nu}\right)= \\
& =-g_{F}^{\beta \rho} \cup_{\rho}{ }^{\alpha \mu}
\end{aligned}
$$

Then the contravariant form of equation (2.3.8.) is

$$
\sqrt{|g|}\left(G^{\alpha \beta}+\kappa_{E} \theta^{\alpha \beta}\right)=-\partial_{\mu}\left(\sqrt{|g|} \kappa_{E} \sigma^{\mu \alpha \beta}\right) .
$$

The antisymmetric part of ${ }_{E} \sigma^{\mu \alpha \beta}, \frac{1}{2}\left({ }_{E} \sigma^{\mu \alpha \beta}-{ }_{E} \sigma^{\mu \beta \alpha}\right)$, is just ${ }_{E} \sigma^{\mu[\alpha \beta]}$ introduced earlier, while the antisymmetric part of (2.3.15.), the contravariant form of (2.3.8.), is just (2.3.12.). Trivially, ${ }_{E} \sigma^{\mu \alpha \beta}={ }_{E} \sigma^{[\mu \alpha] \beta}$ and ${ }_{E} \sigma^{[\mu \alpha \beta]}=0$. However, (2.3.8.) does not determine ${ }_{E} \sigma^{\mu \alpha \beta}$ uniquely: we can add to ${ }_{E} \sigma^{\mu \alpha \beta}$ a total pseudodivergence $\partial_{\nu} V^{\beta \alpha \mu \nu}, V^{\beta \alpha \mu \nu}=V^{\beta \alpha[\mu \nu]}$, such that (2.3.15.) remains the same. We discuss the effect of this freedom in paragraph 2.6.

In the classical Lagrangian theory of matter fields the Belinfante-Rosenfeld combination of $\theta^{\alpha \beta}$ and $\sigma^{\mu \alpha \beta}$ is a gauge invariant tensor field which, moreover, it does not depend on total divergences added to the Lagrangian. It might therefore be interesting to see what is the Belinfante-Rosenfeld combination of the canonical energy-momentum and spin pseudotensors. It is

$$
\begin{aligned}
\sqrt{|g|} \theta_{E} \theta^{\alpha \beta} & +\partial_{\mu}\left(\sqrt{|g|}\left[E_{E} \sigma^{\mu[\alpha \beta]}+{ }_{E} \sigma^{\alpha[\beta \mu]}+{ }_{E} \sigma^{\beta[\alpha \mu]}\right]\right)= \\
& =-\frac{1}{\kappa} \sqrt{|g|} G^{\alpha \beta}+\partial_{\mu}\left(\frac{1}{2 \kappa} g^{\beta \rho}{ }_{F} \cup_{\rho}{ }^{\alpha \mu}+\sqrt{|g|}\left[{ }_{E} \sigma^{\mu[\alpha \beta]}+{ }_{E} \sigma^{\alpha[\beta \mu]}+{ }_{E} \sigma^{\beta[\alpha \mu]}\right]\right)= \\
& =-\frac{1}{\kappa} \sqrt{|g|} G^{\alpha \beta} .
\end{aligned}
$$

Thus, surprisingly enough at first sight, the Belinfante-Rosenfeld combination of the highly coordinate dependent objects ${ }_{E} \theta^{\alpha \beta}$ and ${ }_{E} \sigma^{\mu \alpha \beta}$ is tensorial, i.e. gauge (coordinate) independent; moreover, since the Einstein tensor is an Euler-Lagrange expression, it does not depend on total (pseudo) divergences added to the gravitational Lagrangian. However, these were the most remarkable properties of the Belinfante-Rosenfeld combinations of the canonical energy-momentum and spin tensors in the theory of matter fields. It is interesting that, apart from the factor $\sqrt{|g|}$, this Belinfante-Rosenfeld combination is just the tensor that the covariant analysis at the beginning of the previous section suggested as the gravitational energy-momentum tensor.

For any vector field $\mathbf{K}$ one can form the following pseudocurrent: 


$$
\begin{aligned}
{ }_{E} C^{\mu}[\mathbf{K}]: & ={ }_{E} \theta^{\mu \nu} K_{\nu}+\left({ }_{E} \sigma^{\mu[\alpha \beta]}+{ }_{E} \sigma^{\alpha[\beta \mu]}+{ }_{E} \sigma^{\beta[\alpha \mu]}\right) \partial_{\alpha} K_{\beta}= \\
& ={ }_{E} \theta^{\mu \nu} K_{\nu}+{ }_{E} \sigma^{\mu \alpha \beta} \partial_{\alpha} K_{\beta}= \\
& =-\frac{1}{\kappa} G^{\mu \nu} K_{\nu}+\frac{1}{2 \kappa \sqrt{|g|}} \partial_{\rho}\left(K_{F}^{\beta} \cup_{\beta}{ }^{\mu \rho}\right) .
\end{aligned}
$$

The sum $\sqrt{|g|}\left(\kappa_{E} C^{\mu}[\mathbf{K}]+G^{\mu}{ }_{\nu} K^{\nu}\right)$, being the coordinate divergence of an antisymmetric quantity, is identically pseudoconserved, and $\frac{1}{2} K^{\alpha}{ }_{F} \cup_{\alpha}{ }^{\mu \rho}$ is the corresponding superpotential. Since

$$
\begin{aligned}
\kappa \partial_{\mu}\left(\sqrt{|g|} C^{\mu}[\mathbf{K}]\right) & =-\partial_{\mu}\left(\sqrt{|g|} G^{\mu \nu} K_{\nu}\right)= \\
& =-\frac{1}{2} \sqrt{|g|} G^{\mu \nu} \mathrm{Ł}_{\mathbf{K}} g_{\mu \nu}
\end{aligned}
$$

the pseudocurrent $\sqrt{|g|_{E}} C^{\mu}[\mathbf{K}]$ is pseudoconserved in vacuum, but for general Einstein tensor $\sqrt{|g|_{E}} C^{\mu}[\mathbf{K}]$ is pseudo-conserved iff $\mathbf{K}$ is a Killing symmetry of the geometry. Then ${ }_{E} C^{\mu}[\mathbf{K}]$ and $G^{\mu \nu} K_{\nu}$ are conserved separately. $\sqrt{|g|}{ }_{E} C^{\mu}[\mathbf{K}]$ is pseudoconserved even if $\mathbf{K}$ is only a conformal Killing vector provided the scalar curvature of the spacetime vanishes. This result is in accordance with our physical picture: the sum of the gravitational and matter Noether pseudocurrents is pseudoconserved, and the gravitational and matter pseudocurrents are conserved separately only if they are associated to a symmetry of the "gravitational interaction".

It might be interesting to note that eq.(2.3.17.) can be rewritten in the following form

$$
2 \sqrt{|g|}\left(G^{\mu \nu} K_{\nu}+\kappa_{E} C^{\mu}[\mathbf{K}]\right)=\partial_{\nu}\left(\sqrt{|g|}\left(\nabla^{\mu} K^{\nu}-\nabla^{\nu} K^{\mu}\right)+\frac{1}{\sqrt{|g|}} \partial_{\rho}\left(|g| G^{\mu \nu \gamma \rho} K_{\gamma}\right)\right)
$$

where the first term on the right hand side is the familiar tensorial superpotential of Komar [21]. The vector fields satisfying $\partial_{(\alpha} K_{\beta)}=0$ are thought to have distinguished role in the theory of pseudotensors since the associated ${ }_{E} C^{\mu}[\mathbf{K}]$ are considered to be the energy-momentum and total angular momentum pseudocurrents. In fact, $\partial_{(\mu} K_{\nu)}=0$ has $\frac{1}{2} m(m+1)$ independent solutions: $m$ coordinate translations $K_{\mu}^{\alpha}=\delta_{\mu}^{\alpha}$ ( $\alpha$ is the "name index") and $\frac{1}{2} m(m-1)$ coordinate rotations: $K_{\mu}^{\alpha \beta}=x^{\alpha} \delta_{\mu}^{\beta}-x^{\beta} \delta_{\mu}^{\alpha}$. For the coordinate translations (2.3.17.) is just the contravariant form of the von Freud equation (2.3.11.), but for the coordinate rotation $K_{\mu}^{\alpha \beta}$ (2.3.17.) takes the form

$$
2 \sqrt{|g|}\left(M^{\mu \alpha \beta}+\kappa_{E} \mu^{\mu \alpha \beta}\right)=\partial_{\nu}\left(x^{\alpha} g_{F}^{\beta \rho} \cup_{\rho}^{\mu \nu}-x^{\beta} g^{\alpha \rho} \cup_{\rho}{ }^{\mu \nu}\right),
$$

where $M^{\mu \alpha \beta}:=G^{\mu \nu} K_{\nu}^{\alpha \beta}=G^{\mu \beta} x^{\alpha}-G^{\mu \alpha} x^{\beta}$ and

$$
{ }_{E} \mu^{\mu \alpha \beta}:={ }_{E} C^{\mu}\left[\mathbf{K}^{\alpha \beta}\right]={ }_{E} \theta^{\mu \beta} x^{\alpha}-{ }_{E} \theta^{\mu \alpha} x^{\beta}-2{ }_{E} \sigma^{\mu[\beta \alpha]},
$$

the so-called total angular momentum pseudotensor. One can see that, in fact, ${ }_{E} \sigma^{\mu \alpha \beta}$ plays the role of the spin part of the total angular momentum. The superpotential $K_{\nu}^{\alpha \beta} g^{\nu \gamma}{ }_{F} \cup_{\gamma}{ }^{\mu \rho}$, the right hand side of (2.3.20.), is just the angular momentum superpotential of Bergmann and Thomson [23], introduced in a rather heuristic way.

The equation $\partial_{(\mu} K_{\nu)}=0$ can be thought of as the Killing equation in a local flat geometry $\left(U, \eta_{\alpha \beta}\right)$ where $\left(x^{1}, \ldots, x^{m}\right)$ are Descartes coordinates. To specify a wider class of vector fields $K^{\mu}$ one can relax the Killing equation e.g. to the conformal Killing equation $\partial_{(\mu} K_{\nu)}=\frac{1}{2} \omega \eta_{\mu \nu}$. For $m \neq 2$ its new solutions are the so-called special conformal Killing vectors, or rather 1 -forms: 
$C_{\mu}^{\alpha}=2 x^{\alpha} x^{\beta} \eta_{\beta \mu}-x^{\rho} x^{\gamma} \eta_{\rho \gamma} \delta_{\mu}^{\alpha}$ ( $\alpha$ is the "name index") and the dilatation: $D_{\mu}=x^{\rho} \eta_{\rho \mu}$; and the corresponding pseudocurrents can be called conformal pseudocurrents. Another possible weakening

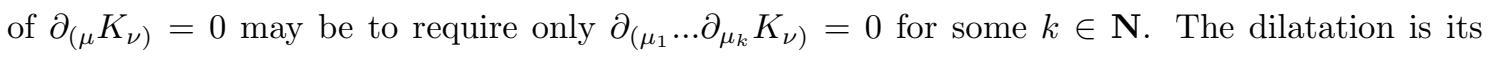
solution for $k=2$ and the special conformal Killing 1 forms for $k=3$.

\subsection{Einstein's pseudotensors in anholonomic description}

In the anholonomic description the gravitational field variables are local basis 1-forms $\left\{\vartheta_{\alpha}^{a}\right\}$ defined on an open domain $U \subset M$, and normalized by

$$
g_{\alpha \beta}=\vartheta_{\alpha}^{a} \vartheta_{\beta}^{b} \eta_{a b},
$$

where $\eta_{a b}$ is a constant matrix (see e.g. [24]). Let $\left\{E_{a}^{\alpha}\right\}$ be local basis vectors on $U$ such that $\left\{E_{a}^{\alpha}\right\}$ and $\left\{\vartheta_{\alpha}^{a}\right\}$ are dual bases:

$$
\vartheta_{\mu}^{a} E_{b}^{\mu}=\delta_{b}^{a}, \vartheta_{\alpha}^{e} E_{e}^{\beta}=\delta_{\alpha}^{\beta} .
$$

The structure functions $c_{r s}^{a}$ on $U$ are defined by

$$
\left[\mathbf{E}_{r}, \mathbf{E}_{s}\right]=c_{r s}^{a} \mathbf{E}_{a} .
$$

Then trivially $c_{r s}^{a}=E_{r}^{\alpha} E_{s}^{\beta}\left(\partial_{\beta} \vartheta_{\alpha}^{a}-\partial_{\alpha} \vartheta_{\beta}^{a}\right)$ and $d \vartheta^{a}=-\frac{1}{2} c_{r s}^{a} \vartheta^{r} \wedge \vartheta^{s}$.

Ricci's rotational coefficients are defined by

$$
\gamma_{r b}^{a}:=\left\langle\vartheta^{a}, \nabla_{\mathbf{E}_{r}} \mathbf{E}_{b}\right\rangle=-\nabla_{\rho} \vartheta_{\beta}^{a} E_{r}^{\rho} E_{b}^{\beta} .
$$

Because of the metric compatibility of the connection $\eta_{a e} \gamma_{r b}^{e}=-\eta_{b e} \gamma_{r a}^{e}$, and because of the vanishing of the torsion

$$
c_{r s}^{a}=\gamma_{r s}^{a}-\gamma_{s r}^{a}
$$

and

$$
\gamma_{r b}^{a}=\frac{1}{2}\left(c_{r b}^{a}+\eta^{a e} c_{e r}^{f} \eta_{f b}+\eta^{a e} c_{e b}^{f} \eta_{f r}\right) .
$$

The components of the curvature and Ricci tensors in the bases $\left\{E_{a}^{\alpha}\right\}$ and $\left\{\vartheta_{\alpha}^{a}\right\}$ are

$$
R_{b c d}^{a}=\gamma_{d b \mid c}^{a}-\gamma_{c b \mid d}^{a}+\gamma_{c r}^{a} \gamma_{d b}^{r}-\gamma_{d r}^{a} \gamma_{c b}^{r}-c_{c d}^{r} \gamma_{r b}^{a}
$$

and

$$
R_{b}^{a}:=\eta^{a e} R_{e b}=\eta^{a e}\left(\gamma_{b e \mid r}^{r}-\gamma_{r e \mid b}^{r}+\gamma_{s r}^{s} \gamma_{b e}^{r}-\gamma_{s b}^{r} \gamma_{r e}^{s}\right),
$$

where the stroke denotes partial directional derivative: $\gamma_{d b \mid c}^{a}:=E_{c}^{\alpha} \partial_{\alpha} \gamma_{d b}^{a}$. The scalar curvature is

$$
R=\eta^{b d}\left(-2 \gamma_{r b \mid d}^{r}+\gamma_{s r}^{s} \gamma_{b d}^{r}-\gamma_{s b}^{r} \gamma_{r d}^{s}\right)
$$

and thus 


$$
\begin{aligned}
\sqrt{|g|} R & =-2 \eta^{r s} \partial_{\mu}\left(\sqrt{|g|} E_{r}^{\mu} \gamma_{k s}^{k}\right)+\sqrt{|g|}\left(\gamma_{r l}^{k} \gamma_{k s}^{l} \eta^{r s}-\gamma_{k l}^{k} \gamma_{r s}^{l} \eta^{r s}\right)= \\
& =-2 \eta^{r s} \partial_{\mu}\left(\sqrt{|g|} E_{r}^{\mu} \gamma_{k s}^{k}\right)+ \\
& +\sqrt{|g|} \eta^{b d}\left(c_{r b}^{r} c_{s d}^{s}-\frac{1}{2} c_{s b}^{r} c_{r d}^{s}-\frac{1}{4} c_{k b}^{r} c_{l d}^{s} \eta^{k l} \eta_{r s}\right) .
\end{aligned}
$$

In the anholonomic description the Lagrangian is chosen to be [24]

$$
\mathcal{L}_{a E}:=\frac{1}{2 \kappa} \sqrt{|g|}\left(c_{r b}^{r} c_{s d}^{s}-\frac{1}{2} c_{s b}^{r} c_{r d}^{s}-\frac{1}{4} c_{k b}^{r} c_{l d}^{s} \eta^{k l} \eta_{r s}\right) \eta^{b d} .
$$

Apart from the $\sqrt{|g|}$ factor, this is scalar, but, of course, not $O(p, q)$-gauge invariant. The canonical energy-momentum pseudotensor derived from $\mathcal{L}_{a E}$ is

$$
\begin{aligned}
2 \kappa \sqrt{|g|_{a E}} t_{\nu}^{\mu}: & =2 \kappa\left(\mathcal{L}_{a E} \delta_{\nu}^{\mu}-\frac{\partial \mathcal{L}_{a E}}{\partial \partial_{\mu} \vartheta_{\rho}^{e}} \partial_{\nu} \vartheta_{\rho}^{e}\right)= \\
& =\sqrt{|g|}\left\{\delta_{b}^{a}\left(\gamma_{r e}^{r} \gamma_{s f}^{s}-\gamma_{s e}^{r} \gamma_{r f}^{s}\right) \eta^{e f}+\right. \\
& \left.+2 \vartheta_{\rho}^{r} E_{s \mid b}^{\rho}\left(\gamma_{r e}^{a} \eta^{e s}+\left(\delta_{e}^{a} \delta_{r}^{s}-\delta_{r}^{a} \delta_{e}^{s}\right) \eta^{e k} \gamma_{l k}^{l}\right)\right\} E_{a}^{\mu} \vartheta_{\nu}^{b} .
\end{aligned}
$$

This energy-momentum pseudotensor can be derived from Goldberg's superpotential [8]:

$$
2 \sqrt{|g|}\left(G_{\beta}^{\alpha}+\kappa_{a E} t^{\alpha}{ }_{\beta}\right)=\partial_{\mu G} \cup_{\beta}{ }^{\alpha \mu},
$$

where

$$
{ }_{G} \cup_{\beta}^{\alpha \mu}:=2 \sqrt{|g|}\left(-\gamma_{b e}^{a} \eta^{e r}+\left(\delta_{b}^{a} \delta_{s}^{r}-\delta_{s}^{a} \delta_{b}^{r}\right) \eta^{s e} \gamma_{k e}^{k}\right) \vartheta_{\beta}^{b} E_{a}^{\alpha} E_{r}^{\mu} .
$$

Trivially, ${ }_{G} \cup_{\beta}{ }^{\alpha \mu}={ }_{G} \cup_{\beta}[\alpha \mu],{ }_{G} \cup_{\rho}{ }^{\alpha \rho}=(m-2)_{a} l^{\alpha}$, where ${ }_{a} l^{\alpha}=-2 \sqrt{|g|} \eta^{a e} E_{a}^{\alpha} \gamma_{r e}^{r}$ is the vector density whose coordinate divergence has been left from $\sqrt{|g|} R$ to obtain the Lagrangian. It might be worth noting that Goldberg's superpotential, apart from $\sqrt{|g|}$, is a tensor field but it, of course, depends on the field of basis 1-forms; i.e. ${ }_{G} \cup_{\beta}{ }^{\alpha \mu}$ is not $O(p, q)$-gauge invariant. ${ }_{G} \cup_{\beta}{ }^{\alpha \mu}$ can be derived from the "square-root" of the Landau-Lifshitz superpotential: If

$$
H_{a b}^{\alpha \beta}:=\frac{1}{\sqrt{2}} \sqrt{|\eta|} \operatorname{det}\left\|\vartheta_{\rho}^{r}\right\|\left(E_{a}^{\alpha} E_{b}^{\beta}-E_{a}^{\beta} E_{b}^{\alpha}\right)
$$

then $H_{a b}^{\alpha \beta}=H_{a b}^{[\alpha \beta]}=H_{[a b]}^{\alpha \beta}, H_{a b}^{\alpha \beta} H_{c d}^{\gamma \delta} \eta^{a c} \eta^{b d}=H^{\alpha \beta \gamma \delta}$ and

$$
{ }_{G} \cup_{\mu}^{\alpha \beta}=g_{\mu \rho}\left(H_{a b}^{\alpha \beta} \eta^{a r} \eta^{b s} \partial_{\nu} H_{r s}^{\rho \nu}+H_{a r}^{\alpha \rho} \eta^{a b} \eta^{r s} \partial_{\nu} H_{b s}^{\beta \nu}-H_{b r}^{\beta \rho} \eta^{b a} \eta^{r s} \partial_{\nu} H_{a s}^{\alpha \nu}\right) .
$$

The contravariant form of ${ }_{a E} t^{\alpha}{ }_{\beta}$ is

$$
\begin{aligned}
\kappa_{a E} \theta^{\alpha \beta}: & =\kappa_{a E} t^{\alpha \beta}+\frac{1}{2 \sqrt{|g|}} \partial_{\mu} g^{\beta \rho}{ }_{G} \cup_{\rho}^{\alpha \mu}= \\
& =\frac{1}{2} g^{\alpha \beta}\left(\gamma_{r b}^{r} \gamma_{s d}^{s}-\gamma_{s b}^{r} \gamma_{r d}^{s}\right) \eta^{b d}+ \\
& +\left(\gamma_{s k}^{r} \gamma_{r l}^{s}-\gamma_{l e}^{r} \gamma_{r k}^{e}-\gamma_{r k}^{r} \gamma_{s l}^{s}+\gamma_{s l}^{r} \gamma_{e f}^{e} \eta_{k r} \eta^{s f}+\gamma_{l k}^{r} \gamma_{e r}^{e}\right) \eta^{k a} \eta^{l b} E_{a}^{\alpha} E_{b}^{\beta}+ \\
& +\vartheta_{\rho}^{b} E_{k \mid e}^{\rho} \eta^{k r}\left(-\gamma_{r s}^{a} \eta^{s e}+\left(\delta_{r}^{a} \delta_{s}^{e}-\delta_{s}^{a} \delta_{r}^{e}\right) \eta^{s l} \gamma_{f l}^{f}\right) E_{a}^{\alpha} E_{b}^{\beta}
\end{aligned}
$$


for which

$$
2 \sqrt{|g|}\left(G^{\alpha \beta}+\kappa_{a E} \theta^{\alpha \beta}\right)=\partial_{\mu}\left(g^{\beta \rho} \cup_{\rho}{ }^{\alpha \mu}\right) .
$$

Neither ${ }_{a E} t^{\alpha \beta}$ nor ${ }_{a E} \theta^{\alpha \beta}$ is symmetric: Taking the antisymmetric part of (2.4.10.) we obtain

$$
2 \kappa \sqrt{|g|}_{a E} \theta^{[\alpha \beta]}=\frac{1}{2} \partial_{\mu}\left(g^{\beta \rho}{ }_{G} \cup_{\rho}^{\alpha \mu}-g^{\alpha \rho}{ }_{G} \cup_{\rho}^{\beta \mu}\right) .
$$

Therefore if we defined $4 \kappa \sqrt{|g|}{ }_{a E} \sigma^{\mu[\alpha \beta]}:=g^{\alpha \rho}{ }_{G} \cup_{\rho}{ }^{\beta \mu}-g^{\beta \rho}{ }_{G} \cup_{\rho}{ }^{\alpha \mu}$ then we would have $\sqrt{|g|}$ ${ }_{a E} \theta^{[\alpha \beta]}=-\partial_{\mu}\left(\sqrt{|g|}_{a E} \sigma^{\mu[\alpha \beta]}\right)$.

The canonical spin pseudotensor is defined by (2.2.3.) and (2.4.4.):

$$
\begin{aligned}
2 \kappa \sqrt{|g|}_{a E} s_{\beta}^{\mu \alpha}: & =2 \kappa \frac{\partial \mathcal{L}_{a E}}{\partial \partial_{\mu} \vartheta_{\rho}^{r}}\left(-\delta_{\rho}^{\alpha} \vartheta_{\beta}^{r}\right)= \\
& =-2 \sqrt{|g|}\left(-\gamma_{b r}^{a} \eta^{r e}+\left(\delta_{b}^{a} \delta_{s}^{e}-\delta_{s}^{a} \delta_{b}^{e}\right) \eta^{s r} \gamma_{k r}^{k}\right) \vartheta_{\beta}^{b} E_{e}^{\mu} E_{a}^{\alpha}= \\
& =-{ }_{G} \cup_{\beta}{ }^{\alpha \mu},
\end{aligned}
$$

i.e. the anholonomic canonical spin pseudotensor is just the Goldberg superpotential. Thus

$$
\sqrt{|g|}\left(G_{\beta}^{\alpha}+\kappa_{a E} t_{\beta}^{\alpha}\right)=-\partial_{\mu}\left(\kappa \sqrt{|g|}{ }_{a E} s_{\beta}^{\mu \alpha}\right)
$$

and hence

$$
\sqrt{|g|}\left(G^{\alpha \beta}+\kappa_{a E} \theta^{\alpha \beta}\right)=-\partial_{\mu}\left(\kappa \sqrt{|g|}_{a E} s^{\mu \alpha \beta}\right) .
$$

(2.4.13.) has just the same structure as (2.3.8.), it is a relation between canonically defined quantities. $a_{E} s^{\mu \alpha}{ }_{\beta}$ is tensor, and (2.4.14.) suggests to define the covariant form of $a_{E} s^{\mu \alpha}{ }_{\beta}$ simply by

$$
{ }_{a E} \sigma^{\mu \alpha \beta}:={ }_{a E} s^{\mu \alpha \beta}=-\frac{1}{2 \kappa \sqrt{|g|}} g^{\beta \rho}{ }_{G} \cup_{\rho}^{\alpha \mu},
$$

and therefore one has

$$
\sqrt{|g|}\left(G^{\alpha \beta}+\kappa_{a E} \theta^{\alpha \beta}\right)=-\partial_{\mu}\left(\kappa \sqrt{|g|}_{a E} \sigma^{\mu \alpha \beta}\right) .
$$

The antisymmetric part of ${ }_{a E} \sigma^{\mu \alpha \beta}$ is just ${ }_{a E} \sigma^{\mu[\alpha \beta]}$ introduced above and the antisymmetric part of (2.4.16.) looks like as the algebraic Belinfante-Rosenfeld equation.

One can form the Belinfante-Rosenfeld combination of ${ }_{a E} \theta^{\alpha \beta}$ and ${ }_{a E} \sigma^{\mu \alpha \beta}$ which, as one could expect, is just $-\frac{1}{\kappa} \sqrt{|g|}$ times the Einstein tensor again.

One can form the Noether pseudocurrent:

$$
\begin{aligned}
{ }_{a E} C^{\mu}[\mathbf{K}]: & ={ }_{a E} \theta^{\mu \nu} K_{\nu}+\left({ }_{a E} \sigma^{\mu[\alpha \beta]}+{ }_{a E} \sigma^{\alpha[\beta \mu]}+{ }_{a E} \sigma^{\beta[\alpha \mu]}\right) \partial_{\alpha} K_{\beta}= \\
& ={ }_{a E} \theta^{\mu \nu} K_{\nu}+{ }_{a E} \sigma^{\mu \alpha \beta} \partial_{\alpha} K_{\beta}= \\
& =-\frac{1}{\kappa} G^{\mu \nu} K_{\nu}+\frac{1}{2 \kappa \sqrt{|g|}} \partial_{\rho}\left(K^{\nu}{ }_{G} \cup_{\nu}{ }^{\mu \rho}\right) .
\end{aligned}
$$


The pseudocurrent $\sqrt{|g|}\left(\kappa_{a E} C^{\mu}[\mathbf{K}]+G^{\mu \nu} K_{\nu}\right)$ is pseudoconserved again; and $\sqrt{|g|}{ }_{a E} C^{\mu}[\mathbf{K}]$ and $G^{\mu \nu} K_{\nu}$ are conserved in themselves iff $K^{\mu}$ is a Killing vector of the spacetime geometry. One can again introduce the angular momentum pseudocurrents just in the same way we have done in the holonomic description.

Since Goldberg's superpotential, apart from the $\sqrt{|g|}$ factor, is a tensor, we can rewrite our equations in covariant form. If

$$
{ }_{G} \vee_{\beta}^{\alpha \mu}:=\frac{1}{\sqrt{|g|}} G \cup_{\beta}^{\alpha \mu}
$$

then (2.4.6.) is equivalent to

$$
\begin{aligned}
2\left(G_{\beta}^{\alpha}+\kappa_{a E} t_{\beta}^{\alpha}\right) & =\partial_{\mu G} \vee_{\beta}^{\alpha \mu}+\frac{1}{\sqrt{|g|}} \partial_{\mu} \sqrt{|g|_{G}} \vee_{\beta}^{\alpha \mu}= \\
& =\nabla_{\mu G} \vee_{\beta}{ }^{\alpha \mu}+\Gamma_{\mu \beta}^{\rho} \vee_{\rho}^{\alpha \mu}
\end{aligned}
$$

Thus if we define

$$
\begin{aligned}
2 \kappa_{A E} \theta^{\alpha}{ }_{\beta}: & =2 \kappa_{a E} t^{\alpha}{ }_{\beta}-\Gamma_{\mu \beta G}^{\rho} \vee_{\rho}{ }^{\alpha \mu}= \\
& =\left\{\delta_{b}^{a}\left(\gamma_{k r}^{k} \gamma_{l s}^{l}-\gamma_{l r}^{k} \gamma_{k s}^{l}\right) \eta^{r s}+2\left(\gamma_{k r}^{a} \gamma_{b s}^{k}-\gamma_{b r}^{a} \gamma_{k s}^{k}\right) \eta^{r s}\right\} E_{a}^{\alpha} \vartheta_{\beta}^{b},
\end{aligned}
$$

which is a tensor field, and we recall that ${ }_{A E} \sigma^{\mu \alpha}{ }_{\beta}:={ }_{a E} \sigma^{\mu \alpha}{ }_{\beta}={ }_{a E} s^{\mu \alpha}{ }_{\beta}$ is also tensor field then we have

$$
G^{\alpha}{ }_{\beta}+\kappa_{A E} \theta_{\beta}^{\alpha}=-\kappa \nabla_{\mu A E} \sigma^{\mu \alpha}{ }_{\beta}
$$

This implies the covariant

$$
\begin{aligned}
A_{E} \theta^{[\alpha \beta]} & =-\nabla_{\mu A E} \sigma^{\mu[\alpha \beta]} \\
\nabla_{\alpha A E} \theta^{\alpha}{ }_{\beta} & =-R_{\beta \rho \mu \nu A E} \sigma^{\rho \mu \nu}
\end{aligned}
$$

Belinfante-Rosenfeld equations. The covariant Belinfante-Rosenfeld combination of ${ }_{A E} \theta^{\alpha \beta}$ and ${ }_{A E} \sigma^{\mu \alpha \beta}$ is $-\frac{1}{\kappa} G^{\alpha \beta}$. The covariant Noether current associated to $\mathbf{K}$ is

$$
\begin{aligned}
{ }_{A E} C^{\mu}[\mathbf{K}]: & ={ }_{A E} \theta^{\mu \nu} K_{\nu}+\left({ }_{A E} \sigma^{\mu[\alpha \beta]}+{ }_{A E} \sigma^{\alpha[\beta \mu]}+{ }_{A E} \sigma^{\beta[\alpha \mu]}\right) \nabla_{\alpha} K_{\beta}= \\
& =-\frac{1}{\kappa} G^{\mu \nu} K_{\nu}+\frac{1}{2 \kappa} \nabla_{\rho}\left(K^{\nu}{ }_{G} \vee_{\nu}{ }^{\mu \rho}\right) .
\end{aligned}
$$

But since

$$
\nabla_{\rho}\left(K^{\nu}{ }_{G} \vee_{\nu}^{\mu \rho}\right)=\partial_{\rho}\left(K^{\nu}{ }_{G} \vee_{\nu}^{\mu \rho}\right)+\Gamma_{\rho \gamma}^{\mu} K^{\nu}{ }_{G} \vee_{\nu}{ }^{\gamma \rho}+\Gamma_{\rho \gamma}^{\rho} K^{\nu}{ }_{G} \vee_{\nu}{ }^{\mu \gamma}=\frac{1}{\sqrt{|g|}} \partial_{\rho}\left(K^{\nu}{ }_{G} \cup_{\nu}^{\mu \rho}\right),
$$

the Noether pseudocurrent ${ }_{a E} C^{\mu}[\mathbf{K}]$ coincides with ${ }_{A E} C^{\mu}[\mathbf{K}]$, and hence it is, in fact, a true vector field. However, ${ }_{A E} \theta^{\mu \nu},{ }_{A E} \sigma^{\mu \alpha \beta}$ and the Noether current ${ }_{A E} C^{\mu}[\mathbf{K}]$ itself are $O(p, q)$ gauge dependent. This gauge dependence is not surprising, since, e.g. in electrodynamics, neither the canonical tensors nor the Noether currents are gauge invariant. 


\subsection{Møller's pseudotensors}

First consider the holonomic description. Now the gravitational field variables are the metric components again but the Lagrangian is Hilbert's second order scalar Lagrangian:

$$
\mathcal{L}_{H}:=\frac{1}{2 \kappa} \sqrt{|g|} R=\mathcal{L}_{E}+\frac{1}{2 \kappa} \partial_{\rho} l^{\rho},
$$

where $\mathcal{L}_{E}$ and $l^{\rho}$ are given by (2.3.1.) and (2.3.2.), respectively. The canonical energy-momentum pseudotensor can be calculated using (2.2.6): Since

$$
\begin{aligned}
\frac{\partial l^{\rho}}{\partial g_{\alpha \beta}} & =\frac{1}{2}\left(l^{\rho} g^{\alpha \beta}-l^{\alpha} g^{\beta \rho}-l^{\beta} g^{\alpha \rho}\right)+ \\
& +\frac{1}{2} \sqrt{|g|}\left(g^{\rho \mu} \Gamma_{\mu \nu}^{\alpha} g^{\beta \nu}+g^{\rho \mu} \Gamma_{\mu \nu}^{\beta} g^{\alpha \nu}-2 \Gamma_{\mu \nu}^{\rho} g^{\mu \alpha} g^{\nu \beta}\right)
\end{aligned}
$$

and

$$
\frac{\partial l^{\rho}}{\partial \partial_{\mu} g_{\alpha \beta}}=\frac{1}{2} \sqrt{|g|}\left(g^{\rho \alpha} g^{\mu \beta}+g^{\rho \beta} g^{\mu \alpha}-2 g^{\rho \mu} g^{\alpha \beta}\right),
$$

the energy-momentum pseudotensor, ${ }_{M} t^{\alpha}{ }_{\beta}$, is

$$
\begin{aligned}
2 \kappa \sqrt{|g|}_{M} t^{\alpha}{ }_{\beta} & =2 \kappa \sqrt{|g|_{E}} t^{\alpha}{ }_{\beta}-\partial_{\rho}\left(\delta_{\beta}^{\rho} l^{\alpha}-\delta_{\beta}^{\alpha} l^{\rho}\right)= \\
& =\sqrt{|g|}\left(R \delta_{\beta}^{\alpha}+g^{\alpha \rho} \Gamma_{\mu \rho, \beta}^{\mu}-g^{\rho \mu} \Gamma_{\rho \mu, \beta}^{\alpha}\right) .
\end{aligned}
$$

Using ${ }_{F} \cup_{\rho}^{\alpha \rho}=(m-2) l^{\alpha}$ and (2.3.5.) one has

$$
2 \sqrt{|g|}\left(G_{\beta}^{\alpha}+\kappa_{M} t^{\alpha}{ }_{\beta}\right)=\partial_{\mu}\left({ }_{F} \cup_{\beta}{ }^{\alpha \mu}-\frac{1}{m-2} F \cup_{\rho}{ }^{\alpha \rho} \delta_{\beta}^{\mu}+\frac{1}{m-2} F \cup_{\rho}^{\mu \rho} \delta_{\beta}^{\alpha}\right) .
$$

However, the superpotential on the right hand side is just Møller's superpotential [20]:

$$
\begin{aligned}
{ }_{M} \cup_{\beta}{ }^{\alpha \mu}: & ={ }_{F} \cup_{\beta}{ }^{\alpha \mu}-\frac{1}{m-2} \delta_{\beta}^{\mu} \cup_{\rho}{ }^{\alpha \rho}+\frac{1}{m-2} \delta_{\beta F}^{\alpha} \cup_{\rho}{ }^{\mu \rho}= \\
& =\sqrt{|g|} g^{\alpha \rho} g^{\mu \nu}\left(\partial_{\rho} g_{\nu \beta}-\partial_{\nu} g_{\rho \beta}\right),
\end{aligned}
$$

and hence we could recover Møller's energy-momentum pseudotensor, originally defined by (2.5.3.) for $m=4$, as the canonical energy-momentum pseudotensor for Hilbert's Lagrangian. The contravariant form of ${ }_{M} t^{\alpha}{ }_{\beta}$ is defined by

$$
\begin{aligned}
2 \kappa \sqrt{|g|}_{M} \theta^{\alpha \beta}: & =2 \kappa \sqrt{|g|}_{M} t^{\alpha \beta}+\partial_{\mu} g^{\beta \rho}{ }_{M} \cup_{\rho}^{\alpha \mu}= \\
& =2 \kappa \sqrt{|g|_{E}} \theta^{\alpha \beta}+\partial_{\mu}\left(g^{\alpha \beta} l^{\mu}-g^{\beta \mu} l^{\alpha}\right),
\end{aligned}
$$

for which

$$
2 \sqrt{|g|}\left(G^{\alpha \beta}+\kappa_{M} \theta^{\alpha \beta}\right)=\partial_{\mu}\left(g^{\beta \rho}{ }_{M} \cup_{\rho}^{\alpha \mu}\right) .
$$

The canonical spin pseudotensor can be calculated from (2.2.8.), (2.3.2.) and (2.3.6.):

$$
\begin{aligned}
2 \kappa \sqrt{|g|}_{M} s_{\beta}^{\mu \alpha} & =2 \kappa{\sqrt{|g|_{E}}}_{s^{\mu \alpha}{ }_{\beta}-\delta_{\beta}^{\alpha} l^{\mu}+\delta_{\beta}^{\mu} l^{\alpha}=} \\
& =\sqrt{|g|}\left\{\delta_{\beta}^{\mu}\left(\Gamma_{\gamma \nu}^{\alpha} g^{\gamma \nu}-g^{\alpha \nu} \Gamma_{\nu \rho}^{\rho}\right)+g^{\mu \alpha} \Gamma_{\rho \beta}^{\rho}+\delta_{\beta}^{\mu} g^{\alpha \rho} \Gamma_{\rho \nu}^{\nu}-2 g^{\alpha \rho} \Gamma_{\rho \beta}^{\mu}\right\} .
\end{aligned}
$$


This implies

$$
\begin{aligned}
2 \kappa \sqrt{|g|}_{M} s^{\mu \alpha}{ }_{\beta}+{ }_{M} \cup_{\beta}{ }^{\alpha \mu} & =2 \kappa \sqrt{|g|}_{E} s^{\mu \alpha}{ }_{\beta}+{ }_{F} \cup_{\beta}{ }^{\alpha \mu}= \\
& =\partial_{\nu}\left(\sqrt{|g|} G^{\alpha \rho \mu \nu} g_{\rho \beta}\right),
\end{aligned}
$$

and hence

$$
\sqrt{|g|}\left(G_{\beta}^{\alpha}+\kappa_{M} t_{\beta}^{\alpha}\right)=-\kappa \partial_{\mu}\left(\sqrt{|g|}_{M} s_{\beta}^{\mu \alpha}\right) .
$$

The structure of this equation is similar to that of eq.(2.3.8.). The contravariant form of ${ }_{M} s^{\mu \alpha}{ }_{\beta}$ is defined by

$$
\begin{aligned}
2 \kappa \sqrt{|g|}_{M} \sigma^{\mu \alpha \beta}: & =2 \kappa \sqrt{|g|}_{M} s^{\mu \alpha \beta}-g^{\beta \rho} \partial_{\nu}\left(\sqrt{|g|} G^{\alpha \gamma \mu \nu} g_{\gamma \rho}\right)= \\
& =-g^{\beta \rho}{ }_{M} \cup_{\rho}{ }^{\alpha \mu},
\end{aligned}
$$

and finally one has

$$
\sqrt{|g|}\left(G^{\alpha \beta}+\kappa_{M} \theta^{\alpha \beta}\right)=-\kappa \partial_{\mu}\left(\sqrt{|g|}_{M} \sigma^{\mu \alpha \beta}\right) .
$$

Its antisymmetric part is a Belinfante-Rosenfeld type equation. (2.5.11.) does not specify ${ }_{M} \sigma^{\mu \alpha \beta}$ uniquely: $\sqrt{|g|}_{M} \sigma^{\mu \alpha \beta}+\partial_{\nu} V^{\beta \alpha \mu \nu}$ also satisfies (2.5.11.) for any $V^{\beta \alpha \mu \nu}=V^{\beta \alpha[\mu \nu]}$.

The Belinfante-Rosenfeld combination of ${ }_{M} \theta^{\alpha \beta}$ and ${ }_{M} \sigma^{\mu \alpha \beta}$ is, essentially, the Einstein tensor again:

$$
\begin{aligned}
\sqrt{|g|}{ }_{M} \theta^{\alpha \beta} & +\partial_{\mu}\left(\sqrt{|g|}\left({ }_{M} \sigma^{\mu[\alpha \beta]}+{ }_{M} \sigma^{\alpha[\beta \mu]}+{ }_{M} \sigma^{\beta[\alpha \mu]}\right)\right)= \\
& =-\frac{1}{\kappa} \sqrt{|g|} G^{\alpha \beta} .
\end{aligned}
$$

The Noether pseudocurrent constructed from the Møller pseudotensors is:

$$
\begin{aligned}
{ }_{M} C^{\mu}[\mathbf{K}]: & ={ }_{M} \theta^{\mu \nu} K_{\nu}+\left({ }_{M} \sigma^{\mu[\alpha \beta]}+{ }_{M} \sigma^{\alpha[\beta \mu]}+{ }_{M} \sigma^{\beta[\alpha \mu]}\right) \partial_{\alpha} K_{\beta}= \\
& =-\frac{1}{\kappa} G^{\mu \nu} K_{\nu}+\frac{1}{2 \kappa \sqrt{|g|}} \partial_{\rho}\left(K^{\beta}{ }_{M} \cup_{\beta}{ }^{\mu \rho}\right) ;
\end{aligned}
$$

and hence $\sqrt{|g|}\left(\kappa_{M} C^{\mu}[\mathbf{K}]+G^{\mu \nu} K_{\nu}\right)$ is always pseudoconserved. In contrast to ${ }_{E} C^{\mu}[\mathbf{K}]$ or ${ }_{a E} C^{\mu}[\mathbf{K}]$, this pseudocurrent contains the second derivative of the metric too. (2.5.12.) can also be written in the form containing Komar's superpotential:

$$
\begin{aligned}
2 \sqrt{|g|}\left(G^{\mu \nu} K_{\nu}+\kappa_{M} C^{\mu}[\mathbf{K}]\right)=\partial_{\nu} & \left(\sqrt{|g|}\left(\nabla^{\mu} K^{\nu}-\nabla^{\nu} K^{\mu}\right)+\right. \\
& \left.+K^{\mu} l^{\nu}-K^{\nu} l^{\mu}+\frac{1}{\sqrt{|g|}} \partial_{\delta}\left(|g| G^{\mu \nu \gamma \delta}\right)\right) .
\end{aligned}
$$

As a consequence the difference of the Noether pseudocurrents built up from Møller's and Einstein's pseudotensors, respectively, is identically pseudoconserved. As is expected, $\sqrt{|g|_{M}} C^{\mu}[\mathbf{K}]$ and $\sqrt{|g|} G^{\mu \nu} K_{\nu}$ are pseudoconserved if $\mathbf{K}$ is a Killing vector.

In the anholonomic description the Lagrangian is $\frac{1}{2 \kappa}$ times the right hand side of eq.(2.4.3.), and hence the canonical energy-momentum pseudotensor, ${ }_{a M} t^{\alpha}{ }_{\beta}$, can be calculated using (2.2.7.) and (2.4.5.): 


$$
\begin{aligned}
2 \kappa \sqrt{|g|}_{a M} t_{\beta}^{\alpha} & =2 \kappa \sqrt{|g|_{a E}} t_{\beta}^{\alpha}+\delta_{\beta}^{\alpha} \partial_{\mu} l^{\mu}-\partial_{\beta} l^{\alpha}+ \\
& +\partial_{\nu}\left(\sqrt{|g|}\left[g^{\alpha \rho} E_{k}^{\nu}-g^{\nu \rho} E_{k}^{\alpha}\right] \partial_{\beta} \vartheta_{\rho}^{k}\right) .
\end{aligned}
$$

This implies

$$
2 \sqrt{|g|}\left(G_{\beta}^{\alpha}+\kappa_{a M} t_{\beta}^{\alpha}\right)=\partial_{\mu a M} \cup_{\beta}^{\alpha \mu}
$$

where

$$
\begin{aligned}
a M \cup_{\beta}{ }^{\alpha \mu} & ={ }_{G} \cup_{\beta}{ }^{\alpha \mu}+\delta_{\beta}^{\alpha} l^{\mu}-\delta_{\beta}^{\mu} l^{\alpha}+\sqrt{|g|}\left(g^{\alpha \nu} E_{k}^{\mu}-g^{\mu \nu} E_{k}^{\alpha}\right) \partial_{\beta} \vartheta_{\nu}^{k}= \\
& =\sqrt{|g|}\left(\left(g^{\alpha \nu} E_{r}^{\mu}-g^{\mu \nu} E_{r}^{\alpha}\right) \partial_{\nu} \vartheta_{\beta}^{r}+g^{\alpha \nu} g^{\mu \rho}\left(\partial_{\nu} \vartheta_{\rho}^{r}-\partial_{\rho} \vartheta_{\nu}^{r}\right) \eta_{r s} \vartheta_{\beta}^{s}\right)= \\
& =\sqrt{|g|} g^{\alpha \nu} g^{\mu \rho}\left(\partial_{\nu} g_{\rho \beta}-\partial_{\rho} g_{\nu \beta}\right)={ }_{M} \cup_{\beta}{ }^{\alpha \mu},
\end{aligned}
$$

and hence

$$
a M t_{\beta}^{\alpha}={ }_{M} t_{\beta}^{\alpha} \text {. }
$$

The canonical spin pseudotensor can be calculated using (2.2.8.) and (2.4.12):

$$
2 \kappa{\sqrt{|g|_{a M}}}^{\mu \alpha}{ }_{\beta}=-{ }_{a M} \cup_{\beta}^{\alpha \mu}+\partial_{\nu}\left(\sqrt{|g|} G^{\alpha \rho \mu \nu} g_{\rho \beta}\right)=2 \kappa \sqrt{|g|}_{M} s_{\beta}^{\mu \alpha} .
$$

Thus both the canonical energy-momentum and spin pseudotensors in the anholonomic description coincide with the corresponding pseudotensors calculated in the holonomic description; and therefore all the quantities built up from ${ }_{M} \theta^{\alpha}{ }_{\beta}$ and ${ }_{M} s^{\mu \alpha}{ }_{\beta}$ coincide with those built up from ${ }_{a M} \theta^{\alpha}{ }_{\beta}$ and ${ }_{a M} s^{\mu \alpha}{ }_{\beta}$.

\subsection{Landau-Lifshitz-Goldberg type pseudotensors}

In the previous three paragraphs we saw that the canonical spin pseudotensors single out superpotentials for the canonical energy-momentum pseudotensors from the mathematically possible collection of superpotentials. Thus although in general the contravariant forms $\theta^{\alpha \beta}$ of the canonical energy-momentum pseudotensors depend not only on $t_{\beta}^{\alpha}$, but the superpotential we use also, the contravariant forms $\theta^{\alpha \beta}$ we defined in (2.3.10.), (2.4.10.) and (2.5.5.) are distinguished because the superpotentials we used are distinguished by the spin pseudotensors. Since the equations (2.3.5.), (2.4.6.) and (2.5.3.), by means of which the contravariant forms $\theta^{\alpha \beta}$ have been defined, contain $t^{\alpha}{ }_{\beta}$ algebraically, $\theta^{\alpha \beta}$ is uniquely defined by $t^{\alpha}{ }_{\beta}$ and the superpotentials. (We do not use here the indexes $E, a E, M, \ldots$ etc., since the formulae we will have hold for any of the three cases we considered in the previous paragraphs.)

However, the contravariant form $\sigma^{\mu \alpha \beta}$ of the canonical spin pseudotensors are not uniquely determined even if we have preferred superpotentials, since the equations (2.3.8.), (2.4.13.) and (2.5.9.), by means of which $\sigma^{\mu \alpha \beta}$ was defined, contain only the divergence of $\sqrt{|g|} s^{\mu \alpha}$. Thus in the definition of $\sigma^{\mu \alpha \beta}$ we have the freedom

$$
\sigma^{\mu \alpha \beta} \longmapsto \hat{\sigma}^{\mu \alpha \beta}:=\sigma^{\mu \alpha \beta}+\frac{1}{2 \kappa \sqrt{|g|}} \partial_{\nu} V^{\beta \alpha \mu \nu},
$$


where $V^{\beta \alpha \mu \nu}=V^{\beta \alpha[\mu \nu]}$ is arbitrary. Although for $V^{\beta \alpha \mu \nu}=0$ the pseudotensors $\sigma^{\mu \alpha \beta}$ are just the contravariant forms of the distinguished superpotentials, supporting our opinion that $V^{\beta \alpha \mu \nu}=0$ is the "right" choice, we should consider the effect of the freedom (2.6.1.).

The Belinfante-Rosenfeld combination of $\theta^{\alpha \beta}$ and $\hat{\sigma}^{\mu \alpha \beta}$ is

$$
\begin{aligned}
\kappa \theta^{\alpha \beta} & +\kappa \partial_{\mu}\left(\sqrt{|g|}\left(\hat{\sigma}^{\mu[\alpha \beta]}+\hat{\sigma}^{\alpha[\beta \mu]}+\hat{\sigma}^{\beta[\alpha \mu]}\right)\right)= \\
& =-\sqrt{|g|} G^{\alpha \beta}-\frac{1}{2} \partial_{\mu} \partial_{\nu}\left(V^{[\alpha \mu][\beta \nu]}+V^{[\beta \mu][\alpha \nu]}\right) .
\end{aligned}
$$

The second term on the right hand side is just the combination by means of which Goldberg's infinite series of Landau-Lifshitz type pseudotensors is defined. Recall [25] that for any fixed $k \in \mathbf{R}$ Goldberg's $2 k$ th symmetric pseudotensor, ${ }_{G} t_{(2 k)}^{\alpha \beta}$, is defined by

$$
|g|^{k+1}\left(G^{\alpha \beta}+\kappa_{G} t_{(2 k)}^{\alpha \beta}\right):=\frac{1}{2} \partial_{\mu} \partial_{\nu}\left(|g|^{k+1} G^{\alpha \mu \beta \nu}\right) .
$$

For $k=0$ this is known as the Landau-Lifshitz pseudotensor [26]: ${ }_{L L} t^{\alpha \beta}:={ }_{G} t_{(0)}^{\alpha \beta}$. Thus for a general $V^{\beta \alpha \mu \nu}$ the Belinfante-Rosenfeld combination is not expected to be tensorial. In fact, rather long but simple calculations show that the Belinfante-Rosenfeld combination can be tensorial only if the second term on the right hand side of (2.6.2.) vanishes. This is equivalent to the existence of a five index quantity $Z^{\alpha \beta \mu \nu \rho}=Z^{(\alpha \beta) \mu \nu \rho}=Z^{\alpha \beta(\mu \nu) \rho}=Z^{\alpha \beta \mu[\nu \rho]}$ for which

$$
V^{[\alpha \mu][\beta \nu]}+V^{[\beta \mu][\alpha \nu]}+V^{[\alpha \nu][\beta \mu]}+V^{[\beta \nu][\alpha \mu]}=\partial_{\rho} Z^{\alpha \beta \mu \nu \rho} .
$$

This gives restrictions for certain irreducible parts of the general $V^{\alpha \beta \mu \nu}$. Thus although the requirement of the tensorial character of the Belinfante-Rosenfeld combination does not rule out completely the freedom (2.6.1.), as for example the left hand side of eq.(2.6.4.) vanishes for a completely skew $V^{\alpha \beta \mu \nu}$, this result gives a partial support of our choice $V^{\alpha \beta \mu \nu}=0$ in the definition of $\sigma^{\mu \alpha \beta}$. For the other natural choice $-\frac{1}{2} \sqrt{|g|} G^{\beta \alpha \mu \nu}$ in the definitions (2.3.8.) and (2.5.9.) the Belinfante-Rosenfeld combination (2.6.2.) is just Goldberg's - 1 th pseudotensor; i.e. ${ }_{G} t_{(-1)}^{\alpha \beta}$ can naturally be recovered as a Belinfante-Rosenfeld combination.

\section{Energy-momentum and spin forms on $L(M)$}

\subsection{Metric connection on $L(M)$}

Let $L(M)$ be the linear frame bundle over $M,\left\{\delta_{i}\right\}, i=1, \ldots, m$, be the standard basis for $\mathbf{R}^{m}$, i.e. $\delta_{i}=\left(0, \ldots, 0,1_{i}, 0, \ldots, 0\right)$ and $\theta=\theta^{i} \delta_{i}$ the canonical $\mathbf{R}^{m}$-valued 1-form on $L(M)$. (For the differential geometric preliminaries see, for example, [27].) The metric $g$ and the volume form $\varepsilon$ of $M$ define a set of functions on $L(M)$. If, for example, $w=\left(p,\left\{\mathbf{E}_{a}\right\}\right) \in L(M)$; i.e. $w$ is a basis $\left\{\mathbf{E}_{a}\right\}$ at $T_{p} M$, then $g_{a b}(w):=g_{p}\left(\mathbf{E}_{a}, \mathbf{E}_{b}\right)$ and $\varepsilon_{a_{1} \ldots a_{m}}(w):=\varepsilon_{p}\left(\mathbf{E}_{a_{1}}, \ldots, \mathbf{E}_{a_{m}}\right)$. One can define $g^{a b}$ and $\varepsilon^{a_{1} \ldots a_{m}}$ too, for which $\varepsilon_{a_{1} \ldots a_{r} e_{r+1} \ldots e_{m}} \varepsilon^{b_{1} \ldots b_{r} e_{r+1} \ldots e_{m}}=(-)^{q}(m-r) ! \delta_{a_{1} \ldots a_{r}}^{b_{1} \ldots b_{r}}$.

For any $r=0,1, \ldots, m$ let $[28,29]$

$$
\Sigma_{a_{1} \ldots a_{r}}:=\frac{1}{(m-r) !} \varepsilon_{a_{1} \ldots a_{r} e_{r+1} \ldots e_{m}} \theta^{e_{r+1}} \wedge \ldots \wedge \theta^{e_{m}} .
$$


It is a tensorial $(m-r)$ form on $L(M)$, transforming according to the $r$ th exterior power of the contragredient representation of $G L(m, \mathbf{R})$. If $r=0$ then this is just $\Sigma:=\frac{1}{m !} \varepsilon_{e_{1} \ldots e_{m}} \theta^{e_{1}} \wedge \ldots \wedge \theta^{e_{m}}$, while for $r=m$ this is the function $\varepsilon_{a_{1} \ldots a_{m}}$. One can easily verify that

$$
\theta^{b} \wedge \Sigma_{a_{1} \ldots a_{r}}=(-)^{r+1} r \delta_{\left[a_{1}\right.}^{b} \Sigma_{\left.a_{2} \ldots a_{r}\right]}
$$

Let $\omega^{a}{ }_{b}$ be a connection 1-form on $L(M)$ compatible with the metric $g$. The structure equations for the torsion $\Xi^{a}$ and curvature 2 -form $\Omega^{a}{ }_{b}$ are

$$
\begin{aligned}
\Xi^{a} & =d \theta^{a}+\omega^{a}{ }_{b} \wedge \theta^{b} \\
\Omega^{a}{ }_{b} & =d \omega^{a}{ }_{b}+\omega^{a}{ }_{e} \wedge \omega^{e}{ }_{b} .
\end{aligned}
$$

If index lowering and rising are defined by $g_{a b}$ and $g^{a b}$, e.g. $\omega_{a b}:=g_{a e} \omega^{e}{ }_{b}$, then the condition of metric compatibility is

$$
d g_{a b}=\omega_{a b}+\omega_{b a} .
$$

This implies $d \varepsilon_{a_{1} \ldots a_{m}}=\varepsilon_{a_{1} \ldots a_{m}} \omega^{e}{ }_{e}$ and, in general,

$$
d \Sigma_{a_{1} \ldots a_{r}}=\Xi^{a} \wedge \Sigma_{a_{1} \ldots a_{r} a}-(-)^{r} r \omega_{\left[a_{1}\right.}^{a} \wedge \Sigma_{\left.a_{2} \ldots a_{r}\right] a} .
$$

The first and the second Bianchi identities are

$$
\begin{aligned}
d \Xi^{a} & =\Omega^{a}{ }_{e} \wedge \theta^{e}-\omega^{a}{ }_{e} \wedge \Xi^{e} \\
d \Omega^{a}{ }_{b} & =\Omega^{a}{ }_{e} \wedge \omega^{e}{ }_{b}-\omega^{a}{ }_{e} \wedge \Omega^{e}{ }_{b} .
\end{aligned}
$$

Because of the metric compatibility, $\Omega_{a b}:=g_{a e} \Omega_{b}^{e}=-\Omega_{b a}$. In this formalism Einstein's tensor, $G^{i}{ }_{j}:=R^{i}{ }_{j}-\frac{1}{2} R \delta_{j}^{i}$, is given by

$$
-\frac{1}{2} \Omega^{a b} \wedge \Sigma_{i a b}=G^{j}{ }_{i} \Sigma_{j}
$$

The curvature tensor can be expressed by horizontal $m$-forms:

$$
\Omega_{b}^{a} \wedge \Sigma_{c d}=R_{b c d}^{a} \Sigma
$$

and hence both the Ricci tensor and the curvature scalar can also:

$$
-\Omega^{a j} \wedge \Sigma_{i a}=R^{j}{ }_{i} \Sigma
$$

and

$$
\begin{aligned}
R \Sigma & =\Omega^{a b} \wedge \Sigma_{a b}= \\
& =d\left(\omega^{a b} \wedge \Sigma_{a b}\right)-\omega^{a}{ }_{e} \wedge \omega^{e b} \wedge \Sigma_{a b}+\omega^{a b} \wedge \Xi^{e} \wedge \Sigma_{a b e}
\end{aligned}
$$

respectively.

A local section $s: U \rightarrow L(M)$ is a field of basis vectors $\left\{\mathbf{E}_{a}\right\}$ on $U$. (More precisely, the vector $\mathbf{E}_{a}$ at $p \in U$ is the element $s(p)\left(\delta_{a}\right)$ of the vector bundle $T(M)$, associated to $s(p) \in L(M)$ and 
$\delta_{a} \in \mathbf{R}^{m}$.) The pull back $s^{*}\left(\theta^{a}\right)$ is a field of basis 1 -forms on $U$, dual to the vector basis. The structure coefficients of the section is the collection $\left\{c_{r s}^{a}\right\}$ of functions defined on $U$ by $\left[\mathbf{E}_{r}, \mathbf{E}_{s}\right]=$ $c_{r s}^{a} \mathbf{E}_{a} . s$ is called coordinate or holonomic section if there is a coordinate system $\left(x^{1}, \ldots, x^{m}\right)$ on $U$ such that $\mathbf{E}_{a}=\frac{\partial}{\partial x^{a}}, a=1, \ldots, m$, and then $s^{*}\left(\theta^{a}\right)=d x^{a} . s$ is a coordinate section iff $c_{r s}^{a}=0$. $s$ is called rigid or anholonomic with respect to the metric $g$ if for the pull backs $\vartheta^{a}:=s^{*}\left(\theta^{a}\right)$ and for some constant matrix $\eta_{a b}$ we have $g=\vartheta^{a} \otimes \vartheta^{b} \eta_{a b}$. The pull back $s^{*}\left(\omega^{a}{ }_{b}\right)$ is a $g l(m, \mathbf{R})$ valued 1-form on $U$, thus it can be expressed in the naturally defined basis of 1-forms $\left\{s^{*}\left(\theta^{a}\right)\right\}$ : $s^{*}\left(\omega^{a}{ }_{b}\right)=\omega_{r b}^{a} s^{*}\left(\theta^{r}\right)$. If $s$ is a coordinate section then $\Gamma_{r b}^{a}$, defined by $s^{*}\left(\omega^{a}{ }_{b}\right)=\Gamma_{r b}^{a} d x^{r}$, are the usual Christoffel symbols; while if $s$ is a rigid section then $\gamma_{r b}^{a}$, defined by $s^{*}\left(\omega^{a}{ }_{b}\right)=\gamma_{r b}^{a} \vartheta^{r}$, are the Ricci rotation coefficients. Twice the pull back of the curvature form is just the curvature tensor: $2 s^{*}\left(\Omega_{b}^{a}\right)=R_{b r s}^{a} s^{*}\left(\theta^{r}\right) \wedge s^{*}\left(\theta^{s}\right)$. Since the pull back $s^{*}(\Sigma)$ is the volume form on $M$, the pull back of $\frac{1}{2 \kappa} \Omega^{a b} \wedge \Sigma_{a b}$ gives Hilbert's Lagrangian. It is given in the holonomic/anholonomic description if $s$ is a holonomic/anholonomic section. The pull back of $d\left(\omega^{a b} \wedge \Sigma_{a b}\right)$ gives the total divergence left from Hilbert's Lagrangian, thus Einstein's Lagrangian in the holonomic/anholonomic description is the pull back of $-\frac{1}{2 \kappa} \omega^{a}{ }_{e} \wedge \omega^{e b} \wedge \Sigma_{a b}$ along a holonomic/anholonomic section of $L(M)$.

\subsection{Sparling type forms on $L(M)$}

Let us define the Nester-Witten form as

$$
u_{i}:=-\frac{1}{2} \omega^{a b} \wedge \Sigma_{i a b}
$$

(This form appeared first in its spinorial form by means of which Nester [30] could give a simple proof of Witten's energy positivity theorem [31]. In its tensorial form $u_{i}$ was introduced by Sparling [28]; and for $m$ dimensions $u_{i}$ was defined in [29].) $u_{i}$ is an $\mathbf{R}^{m *}$-valued pseudotensorial $(m-2)$ form which transforms according to the contragredient representation of $G L(m, \mathbf{R})$. Its exterior derivative is

$$
d u_{i}=-\frac{1}{2} \Omega^{a b} \wedge \Sigma_{i a b}+\frac{1}{2} \Xi^{c} \wedge \omega^{a b} \wedge \Sigma_{i a b c}+t_{i},
$$

where

$$
t_{i}:=-\frac{1}{2}\left(\omega^{e}{ }_{i} \wedge \omega^{a b} \wedge \Sigma_{e a b}+\omega^{a}{ }_{e} \wedge \omega^{e b} \wedge \Sigma_{i a b}\right)
$$

is Sparling's $(m-1)$-form [28,29]. What is interesting here is the structure of the right hand side of eq.(3.2.2.): the curvature appears only through the Einstein tensor. $t_{i}$ is only pseudotensorial, transforming according to the contragredient representation of $G L(m, \mathbf{R})$ [32]. The importance of $u_{i}$ and $t_{i}$ is shown by the following theorem, due to Sparling [28] for the vacuum case and Dubois-Violette and Madore [29] for the general case:

Theorem 3.2.1.:

For any $\mathbf{R}^{m *}$-valued horizontal $(m-1)$ form $T_{i}$ satisfying $d T_{i}-\omega^{e}{ }_{i} \wedge T_{e}=0$ and $\kappa \in \mathbf{R}$ the following statements are equivalent:

(1) $\omega^{a}{ }_{b}$ is torsion free, $\Xi^{a}=0$, and $\frac{1}{2} \Omega^{a b} \wedge \Sigma_{i a b}+\kappa T_{i}=0$;

(2) $\kappa T_{i}+t_{i}=d u_{i}$;

(3) $d\left(\kappa T_{i}+t_{i}\right)=0$. 
This theorem gives an alternative formulation of Einstein's theory: a metric connection on $L(M)$ is torsion free and satisfies Einstein's equations with matter energy momentum tensor $T^{j}{ }_{i}$, defined by $T_{i}=: T^{j}{ }_{i} \Sigma_{j}$, iff the Sparling and the Nester-Witten forms satisfy condition (2); which is equivalent to the Sparling form to satisfy condition (3) above. In Einstein's theory (3) looks like as a conservation equation, while (2) tells us the "superpotential" for the conserved quantity $\kappa T_{i}+t_{i}$ : it is just the Nester-Witten form. But since these quantities are defined in $L(M)$ instead of $M$, moreover $t_{i}$ and $u_{i}$ are only pseudotensorial forms, the exterior equations (2) and (3) yield equations in $M$ only if we pull them back along a local section of $L(M)$. In fact, Frauendiener [32] calculated the pull back of $u_{i}$ and $d u_{i}$ along a coordinate section of $L(M)$. Here we first repeat his calculation along a general section and then specialize $s$ to be a coordinate and then a rigid section. Then we pull back various forms of Sparling's equation (2) above and we will recover a number of energy-momentum pseudotensors and superpotentials we have considered.

Since $T_{i}$ is horizontal it has the form $T^{j}{ }_{i} \Sigma_{j}$, thus the pull back of $T_{i}$ is independent of the section: If $\left(x^{1}, \ldots, x^{m}\right)$ is any coordinate system on $U$ then

$$
s^{*}\left(T_{i}\right)=E_{i}^{\beta} T^{\mu}{ }_{\beta} \frac{1}{(m-1) !} \sqrt{|g|} \epsilon_{\mu \gamma_{2} \ldots \gamma_{m}} d x^{\gamma_{2}} \wedge \ldots \wedge d x^{\gamma_{m}} .
$$

The pull back of the Nester-Witten form along a general section $s: U \rightarrow L(M)$ is

$$
\begin{aligned}
s^{*}\left(u_{i}\right) & =-\frac{1}{2} \omega_{r e}^{a} g^{e b} s^{*}\left(\theta^{r} \wedge \Sigma_{i a b}\right)= \\
& =-\frac{1}{2}\left(\omega_{i e}^{[a} g^{b] e}+\delta_{i}^{[a} \omega_{r s}^{b]} g^{r s}-\delta_{i}^{[a} g^{b] e} \omega_{r e}^{r}\right) s^{*}\left(\Sigma_{a b}\right) .
\end{aligned}
$$

If $s$ is a coordinate section and $\left(x^{1}, \ldots, x^{m}\right)$ is the corresponding coordinate system on $U$ (and hence there is no difference between the Greek and Latin indexes), then

$$
\begin{aligned}
s^{*}\left(u_{i}\right) & =\frac{1}{4 \sqrt{|g|}} F \cup_{i}^{a b} s^{*}\left(\Sigma_{a b}\right)= \\
& =\frac{1}{4}{ }_{F} \cup_{i}^{a b} \frac{1}{(m-2) !} \epsilon_{a b e_{3} \ldots e_{m}} d x^{e_{3}} \wedge \ldots \wedge d x^{e_{m}},
\end{aligned}
$$

where $\cup_{i} \cup^{a b}$ is von Freud's superpotential given by (2.3.4.). If $s$ is a rigid section and $\left(x^{1}, \ldots, x^{m}\right)$ is a coordinate system on $U$, then

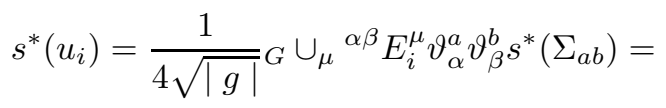

$$
\begin{aligned}
& =\frac{1}{4} E_{i G}^{\mu} \vee_{\mu}{ }^{\alpha \beta} s^{*}\left(\Sigma_{\alpha \beta}\right),
\end{aligned}
$$

where ${ }_{G} \cup_{\mu}^{\alpha \beta}$ is Goldberg's superpotential given by (2.4.7.). Thus, apart from the factor $(2(m-2)$ ! $\sqrt{|g|})^{-1}$, the pull backs of $u_{i}$ are the dual of the von Freud and the Goldberg superpotentials, respectively. If $u_{i}{ }^{a b}$ is defined by $s^{*}\left(u_{i}\right)=u_{i}{ }^{a b} s^{*}\left(\Sigma_{a b}\right)$ then the pull back of the exterior derivative $d u_{i}$ can easily be calculated:

$$
\begin{aligned}
s^{*}\left(d u_{i}\right) & =d s^{*}\left(u_{i}\right)=d u_{i}{ }^{a b} \wedge s^{*}\left(\Sigma_{a b}\right)+u_{i}{ }^{a b} s^{*}\left(d \Sigma_{a b}\right)= \\
& =-2\left(E_{a}^{\mu} \partial_{\mu} u_{i}{ }^{a e}+u_{i}{ }^{a b} \omega_{a b}^{e}+u_{i}{ }^{a e} \omega_{r a}^{r}\right) s^{*}\left(\Sigma_{e}\right) .
\end{aligned}
$$

If $s$ is a coordinate section then $E_{a}^{\mu}=\delta_{a}^{\mu}$ and, using ${ }_{F} \cup_{i}{ }^{a e}={ }_{F} \cup_{i}[a e]$, one has 


$$
\begin{aligned}
s^{*}\left(d u_{i}\right) & =-2\left(\partial_{a} u_{i}^{a e}+\Gamma_{r a}^{r} u_{i}^{a e}\right) s^{*}\left(\Sigma_{e}\right)= \\
& =\frac{1}{2} \partial_{a F} \cup_{i}^{j a} \frac{1}{(m-1) !} \epsilon_{j e_{2} \ldots e_{m}} d x^{e_{2}} \wedge \ldots \wedge d x^{e_{m}} .
\end{aligned}
$$

Comparing (3.2.4.) and (3.2.9) with (2.3.5.) one can expect that the pull back of the Sparling form is essentially Einstein's canonical energy-momentum pseudotensor $[32,33]$. In fact, the pull back of Sparling's form along a general section $s$ is

$$
\begin{aligned}
& s^{*}\left(t_{i}\right)=-\frac{1}{2}( \delta_{i}^{j}\left(\omega_{e k}^{e} \omega_{r s}^{k}-\omega_{r k}^{e} \omega_{e s}^{k}\right) g^{r s}+g^{j k} \omega_{s k}^{s} \omega_{r i}^{r}-g^{j k} \omega_{i k}^{l} \omega_{e l}^{e}-\omega_{e i}^{e} \omega_{r s}^{j} g^{r s}-\omega_{r i}^{j} \omega_{e s}^{e} g^{r s}+ \\
&\left.+\omega_{r k}^{j} \omega_{i s}^{k} g^{r s}+\omega_{k r}^{j} \omega_{s i}^{k} g^{r s}+\left(\omega_{k i}^{j}-\omega_{i k}^{j}\right) \omega_{r s}^{k} g^{r s}+\left(\omega_{i l}^{k}-\omega_{l i}^{k}\right) \omega_{k r}^{l} g^{r j}\right) s^{*}\left(\Sigma_{j}\right)
\end{aligned}
$$

which, if $s$ is a coordinate section, takes the following form

$$
s^{*}\left(t_{i}\right)=\kappa_{E} t^{j}{ }_{i} s^{*}\left(\Sigma_{j}\right)=\kappa_{E} t^{j}{ }_{i} \frac{1}{(m-1) !} \sqrt{|g|} \epsilon_{j e_{2} \ldots e_{m}} d x^{e_{2}} \wedge \ldots \wedge d x^{e_{m}} .
$$

Thus von Freud's equation, given in a coordinate system $\left(x^{1}, \ldots, x^{m}\right)$, is just the pull back of Sparling's equation along the coordinate section associated to $\left(x^{1}, \ldots, x^{m}\right)$. Similarly, the pull back of the conservation equation (3) of Theorem 3.2.1. is just the pseudo divergence equation $\partial_{\alpha}\left(\sqrt{|g|}\left[G^{\alpha}{ }_{\beta}+\kappa_{E} t^{\alpha}{ }_{\beta}\right]\right)=0$. If $s$ is a rigid section then

$$
\begin{aligned}
s^{*}\left(d u_{i}\right) & =-2\left(E_{a}^{\mu} \partial_{\mu} u_{i}{ }^{a e}+u_{i}{ }^{a b} \gamma_{a b}^{e}+u_{i}{ }^{a e} \gamma_{r a}^{r}\right) s^{*}\left(\Sigma_{e}\right)= \\
& =\frac{1}{2 \sqrt{|g|}}\left(E_{i}^{\mu} \vartheta_{\nu}^{e} \partial_{\alpha G} \cup_{\mu}{ }^{\nu \alpha}+{ }_{G} \cup_{\rho}{ }^{\alpha \mu} \vartheta_{\alpha}^{e} \vartheta_{\mu}^{r} E_{i \mid r}^{\rho}\right) s^{*}\left(\Sigma_{e}\right)= \\
& =\frac{1}{2} \nabla_{\rho}\left(E_{i}^{\mu}{ }_{G} \vee_{\mu}{ }^{\nu \rho}\right) s^{*}\left(\Sigma_{\nu}\right),
\end{aligned}
$$

which is tensorial, and the pull back of Sparling's form is

$$
\begin{aligned}
s^{*}\left(t_{i}\right) & =\frac{1}{2}\left(\delta_{i}^{j}\left(\gamma_{e s}^{e} \gamma_{k r}^{k}-\gamma_{k r}^{e} \gamma_{e s}^{k}\right) \eta^{r s}-\right. \\
& \left.-2 \eta^{j k} \gamma_{r k}^{r} \gamma_{s i}^{s}+2\left(\gamma_{r i}^{j}-\gamma_{i r}^{j}\right) \gamma_{k s}^{k} \eta^{r s}+2 \eta^{j r} \gamma_{k r}^{l}\left(\gamma_{l i}^{k}-\gamma_{i l}^{k}\right)\right) s^{*}\left(\Sigma_{j}\right)= \\
& =\left(\kappa_{a E} t_{\nu}^{\mu} \vartheta_{\mu}^{j} E_{i}^{\nu}+\frac{1}{2 \sqrt{|g|}} \cup_{\rho}{ }^{\alpha \mu} \vartheta_{\alpha}^{j} \vartheta_{\mu}^{r} E_{i \mid r}^{\rho}\right) s^{*}\left(\Sigma_{j}\right)= \\
& =\left(\kappa_{A E} \theta^{\mu}{ }_{\nu} E_{i}^{\nu}+\frac{1}{2} G \vee_{\nu}^{\mu \rho} \nabla_{\rho} E_{i}^{\nu}\right) s^{*}\left(\Sigma_{\mu}\right),
\end{aligned}
$$

which is also tensorial. Thus although the components of the pull back of Sparling's form deviates from $\kappa_{a E} t^{j}{ }_{i}$ and from $\kappa_{A E} \theta^{j}{ }_{i}$ too, the pull back of the Sparling equation along a rigid section $s$ of $L(M)$ is just equation (2.4.6.) calculated in the rigid frame determined by $s$. Thus the various energy-momentum pseudotensors are not simply pull backs of a single geometric object, e.g. $t_{i}$, along various sections.

Let us define the contravariant form of the Nester-Witten form simply by $u^{i}:=g^{i j} u_{j}$. Then by (3.1.5.) condition (2) of Theorem 3.2.1. (i.e. Sparling's equation) takes the form

$$
d u^{i}=d g^{i j} \wedge u_{j}+g^{i j} d u_{i}=\kappa T^{i}+\Theta^{i}
$$

where 


$$
\Theta^{i}:=t^{i}-\left(\omega^{i j}+\omega^{j i}\right) \wedge u_{j} .
$$

Obviously, the pull back of $u^{i}$ along coordinate and rigid sections are essentially the contravariant form of von Freud's superpotential: $\frac{1}{4 \sqrt{|g|}} g^{i e}{ }_{F} \cup_{e}{ }^{a b} s^{*}\left(\Sigma_{a b}\right)$ and Goldberg's superpotential: $\frac{1}{4} \eta^{i e} E_{e G}^{\mu} \vee_{\mu}{ }^{\alpha \beta} s^{*}\left(\Sigma_{\alpha \beta}\right)$, respectively. Thus one may expect that the pull back of (3.2.14.) along a coordinate and a rigid section is (2.3.11.) and (2.4.11.), respectively. In fact, the pull back of $d u^{i}$ along a general section is

$$
s^{*}\left(d u^{i}\right)=s^{*}\left(d u_{j}\right) g^{j i}-2\left(\omega_{r s}^{i} g^{s j}+\omega_{r s}^{j} g^{s i}\right) u_{j}^{r e} s^{*}\left(\Sigma_{e}\right),
$$

while if $\tau^{j}{ }_{i}$ is defined by $s^{*}\left(t_{i}\right)=: \tau^{j}{ }_{i} s^{*}\left(\Sigma_{j}\right)$ then

$$
s^{*}\left(\Theta^{i}\right)=\left(\tau^{e}{ }_{k} g^{k i}-2\left(\omega_{r s}^{i} g^{s j}+\omega_{r s}^{j} g^{s i}\right) u_{j}{ }^{r e}\right) s^{*}\left(\Sigma_{e}\right) .
$$

If $s$ is a coordinate section then $s^{*}\left(\Theta^{i}\right)$ gives the contravariant form of Einstein's energy-momentum (i.e. Bergmann's) pseudotensor:

$$
s^{*}\left(\Theta^{i}\right)=\kappa_{E} \theta^{j i} s^{*}\left(\Sigma_{j}\right) ;
$$

but its pull back along a rigid section is not exactly $\kappa_{A E} \theta^{j i}$ :

$$
\begin{aligned}
s^{*}\left(\Theta^{i}\right) & =s^{*}\left(t_{e}\right) \eta^{e i}= \\
& =\left(\kappa_{A E} \theta^{\alpha \beta} \vartheta_{\beta}^{i}+\frac{1}{2}{ }_{G} \vee_{\rho}^{\alpha \mu} \nabla_{\mu} E_{k}^{\rho} \eta^{k i}\right) s^{*}\left(\Sigma_{\alpha}\right) .
\end{aligned}
$$

Let us define the dual form of the Nester-Witten form by

$$
u_{e_{2} \ldots e_{m}}:=u^{e} \varepsilon_{e e_{2} \ldots e_{m}},
$$

and let $T_{e_{2} \ldots e_{m}}:=T^{e} \varepsilon_{e e_{2} \ldots e_{m}}$. Then

$$
d u_{e_{2} \ldots e_{m}}=\kappa T_{e_{2} \ldots e_{m}}+\Theta_{e_{2} \ldots e_{m}},
$$

where

$$
\Theta_{e_{2} \ldots e_{m}}:=\left(t^{e}+\left(g^{e f} \omega^{k}{ }_{k}-\omega^{e f}-\omega^{f e}\right) \wedge u_{f}\right) \varepsilon_{e e_{2} \ldots e_{m}} .
$$

The pull back of $u_{e_{2} \ldots e_{m}}$ along a coordinate section is

$$
\begin{aligned}
s^{*}\left(u_{e_{2} \ldots e_{m}}\right) & =\frac{1}{4 \sqrt{|g|} F} \cup_{f}^{a b} g^{f e} \varepsilon_{e e_{2} \ldots e_{m}} s^{*}\left(\Sigma_{a b}\right)= \\
& =\frac{1}{4} \partial_{r}\left(|g| G^{a b e r}\right) \epsilon_{e e_{2} \ldots e_{m}} \frac{1}{(m-2) !} \epsilon_{a b f_{3} \ldots f_{m}} d x^{f_{3}} \wedge \ldots \wedge d x^{f_{m}},
\end{aligned}
$$

and hence 


$$
\begin{aligned}
s^{*}\left(d u_{e_{2} \ldots e_{m}}\right) & =\frac{1}{2} \partial_{r} \partial_{s}\left(|g| G^{\text {fres }}\right) \frac{1}{(m-1) !} \epsilon_{e e_{2} \ldots e_{m}} \epsilon_{f f_{2} \ldots f_{m}} d x^{f_{2}} \wedge \ldots \wedge d x^{f_{m}}= \\
& =\frac{1}{2|g|} \partial_{r} \partial_{s}\left(|g| G^{f r e s}\right) \frac{1}{(m-1) !} \varepsilon_{e e_{2} \ldots e_{m}} \varepsilon_{f f_{2} \ldots f_{m}} d x^{f_{2}} \wedge \ldots \wedge d x^{f_{m}} .
\end{aligned}
$$

Since the pull back of $d u_{e_{2} \ldots e_{m}}$ is the double dual of the symmetric object defining the LandauLifshitz pseudotensor, one may expect that the pull back of $\Theta_{e_{2} \ldots e_{m}}$ is just the double dual of the Landau-Lifshitz pseudotensor. In fact, the pull back of (3.2.18.) along a coordinate section is

$$
s^{*}\left(\Theta_{e_{2} \ldots e_{m}}\right)=\kappa_{L L} t^{f e} \varepsilon_{e e_{2} \ldots e_{m}} s^{*}\left(\Sigma_{f}\right) .
$$

One can take various forms of the Nester-Witten form and hence the Sparling equation, and one can then pull them back along various local sections of $L(M)$, yielding different superpotentials and pseudotensors derived from Einstein's first order Lagrangian. However, the mathematical content of all these quantities is the same: it is what Sparling's equation describes. From physical points of view, however, these may have different significance: For example if $K_{\beta}$ is any vector field satisfying $\partial_{(\alpha} K_{\beta)}=0$ then for the Landau-Lifshitz pseudotensor we have

$$
\partial_{\alpha}\left(|g|\left(G^{\alpha \beta}+\kappa_{L L} t^{\alpha \beta}\right) K_{\beta}\right)=\frac{1}{2} \partial_{\mu} \partial_{\nu} H^{\alpha \mu \beta \nu} \partial_{(\alpha} K_{\beta)}=0 .
$$

What is (globally) conserved here is therefore the integral

$$
\frac{1}{(m-1) !} \int \sqrt{|g|}\left(G^{\alpha \beta}+\kappa_{L L} t^{\alpha \beta}\right) K_{\beta} \sqrt{|g|} \epsilon_{\alpha \gamma_{2} \ldots \gamma_{m}} d x^{\gamma_{2}} \wedge \ldots \wedge d x^{\gamma_{m}}
$$

for an $(m-1)$ dimensional submanifold. However this is not the energy-momentum of the matter + gravity system even if $\partial_{\alpha} K_{\beta}=0$, since we have an extra $\sqrt{|g|}$ coefficient not only in front of the gravitational part, but in front of the matter part also. If the extra $\sqrt{|g|}$ factor were in front of the gravitational term only but the matter term had the right coefficient then the matter part could be interpreted e.g. as energy-momentum or angular momentum and, in contrast to the strange feature of the gravitational part, would suggest the interpretation of the gravitational part also. The result would be surprising but acceptable [25]. Thus it is hard to interpret these conserved quantities, in contrast to the integral of the Noether pseudocurrents built up e.g. from Einstein's pseudotensors. Moreover if $\mathbf{K}$ is a Killing vector of the geometry then in general the pseudocurrent $|g|\left(G^{\alpha \beta}+\kappa_{L L} t^{\alpha \beta}\right) K_{\beta}$ is not the sum of separately conserved (pseudo) currents, while the Noether pseudo currents built up e.g. from Einstein's pseudotensors are. Perhaps the Landau-Lifshitz pseudocurrents above should be completed by spin parts, but, since the LandauLifshitz pseudotensor is not a canonical pseudotensor, it is not a priori clear how these spin parts should be defined. At the end of the next paragraph we return to this question and construct the missing spin part.

\subsection{The spin form on $L(M)$}

In this paragraph we would like to recover the canonical spin pseudotensors of Einstein's theory as pull backs of a single differential form on $L(M)$, and we would like to interpret this form as the geometric object describing the spin content of gravity. However, there may be different forms $((m-1)$ forms and $(m-2)$ forms) on $L(M)$ whose pull backs yield the same pseudotensors. (For 
example $-u_{i}$ is such an $(m-2)$ form.) But the spin pseudotensors satisfy Belinfante-Rosenfeld equations, thus, in addition, we want to have an exterior differential equation on $L(M)$ whose pull backs are just the Belinfante-Rosenfeld equations.

The quantities in the Belinfante-Rosenfeld equations have two free indexes, thus the spin form would have two free indexes too. Since the spin pseudotensors are three index quantities and we would like to recover them as the duals of the components of the pull backs (as in the case of Sparling's form), the spin form must be an $(m-1)$ form. Thus minus the Nester-Witten form is not the spin form we are searching for. However, the fact that the pull backs of $-u_{i}$ yield the canonical spin pseudotensors suggests to define the spin form by

$$
\sigma^{j}{ }_{i}:=\theta^{j} \wedge u_{i}=\frac{1}{2} \omega^{a b} \wedge\left(\delta_{l}^{j} \Sigma_{a b}+\delta_{a}^{j} \Sigma_{b i}-\delta_{b}^{j} \Sigma_{a i}\right) .
$$

This is a $g l(m, \mathbf{R})$-valued pseudotensorial $(m-1)$ form on $L(M)$ of type ad $G L(m, \mathbf{R})$. It is interesting that its trace,

$$
\sigma^{i}{ }_{i}=\frac{1}{2}(m-2) \omega^{a b} \wedge \Sigma_{a b},
$$

is $\kappa(m-2)$ times the $(m-1)$ form whose exterior derivative has been dropped from $\frac{1}{2 \kappa} R \Sigma$ to obtain the $m$-form describing Einstein's Lagrangian. The pull back of $\sigma^{j}{ }_{i}$ along a local section $s: U \rightarrow L(M)$ is:

$$
\begin{aligned}
s^{*}\left(\sigma^{j}{ }_{i}\right)= & \frac{1}{2}\left(\delta_{i}^{j}\left(\omega_{r s}^{e} g^{r s}-g^{e s} \omega_{r s}^{r}\right)+\right. \\
& \left.+\delta_{i}^{e}\left(\omega_{r s}^{r} g^{s j}-\omega_{r s}^{j} g^{r s}\right)-\delta_{i}^{r}\left(\omega_{r s}^{e} g^{s j}-\omega_{r s}^{j} g^{s e}\right)\right) s^{*}\left(\Sigma_{e}\right) .
\end{aligned}
$$

Thus if $s$ is a coordinate section (when there is no difference between Latin and Greek indexes), then

$$
\begin{aligned}
s^{*}\left(\sigma^{j}{ }_{i}\right) & =\kappa_{E} \sigma^{e j}{ }_{i} s^{*}\left(\Sigma_{e}\right)= \\
& =\kappa_{E} \sigma^{e j}{ }_{i} \frac{1}{(m-1) !} \sqrt{|g|} \epsilon_{e e_{2} \ldots e_{m}} d x^{e_{2}} \wedge \ldots \wedge d x^{e_{m}},
\end{aligned}
$$

while if $s$ is a rigid section then

$$
\begin{aligned}
s^{*}\left(\sigma^{j}{ }_{i}\right) & =\kappa \vartheta_{\alpha}^{j} E_{i a E}^{\beta} \sigma^{\mu \alpha}{ }_{\beta} \vartheta_{\mu}^{e} s^{*}\left(\Sigma_{e}\right)= \\
& =\kappa \vartheta_{\alpha}^{j} E_{i a E}^{\beta} \sigma^{\mu \alpha} \frac{1}{(m-1) !} \sqrt{|g|} \epsilon_{\mu \gamma_{2} \ldots \gamma_{m}} d x^{\gamma_{2}} \wedge \ldots \wedge d x^{\gamma_{m}}
\end{aligned}
$$

The form $\sigma^{j}{ }_{i}$ therefore seems to be a good candidate to be the spin form we search for.

For a moment let us consider general, not necessarily torsion free, metric connections. Then, using (3.1.2.), (3.1.3.) and the general Sparling equation (3.2.2.), we have

$$
\begin{aligned}
-d \sigma^{j}{ }_{i} & =-d \theta^{j} \wedge u_{i}+\theta^{j} \wedge d u_{i}= \\
& =\frac{1}{2} \Xi^{c} \wedge \omega^{a b} \wedge\left(\delta_{i}^{j} \Sigma_{a b c}+\delta_{a}^{j} \Sigma_{i c b}+\delta_{b}^{j} \Sigma_{i a c}\right)+G^{j}{ }_{i} \Sigma+t^{j}{ }_{i},
\end{aligned}
$$

where 


$$
\begin{aligned}
t^{j}{ }_{i}: & =\theta^{j} \wedge t_{i}+\omega^{j}{ }_{e} \wedge \theta^{e} \wedge u_{i}= \\
& =-\frac{1}{2}\left(\delta_{i}^{j} \omega^{a}{ }_{e} \wedge \omega^{e b} \wedge \Sigma_{a b}+\omega^{a}{ }_{i} \wedge\left(\omega^{b j}-\omega^{j b}\right) \wedge \Sigma_{a b}+\omega^{a}{ }_{e} \wedge\left(\omega^{e j}+\omega^{j e}\right) \wedge \Sigma_{i a}\right) .
\end{aligned}
$$

Using eq.(3.3.5.) and the fact that $\Sigma$ is horizontal one can prove easily the next theorem.

\section{Theorem 3.3.1.:}

Let $T_{i}=T^{j}{ }_{i} \Sigma_{j}$ be any horizontal $\mathbf{R}^{m *}$-valued $(m-1)$ form on $L(M), \kappa \in \mathbf{R}$ and $m \neq 2$. Then the following statements are equivalent:

(1) $\omega^{a}{ }_{b}$ is torsion free, $\Xi^{e}=0$, and $G^{j}{ }_{i}=\kappa T^{j}{ }_{i}$,

(2) $\kappa T^{j}{ }_{i} \Sigma+t^{j}{ }_{i}=-d \sigma^{j}{ }_{i}$.

Thus the connections satisfying Einstein's theory can be characterized in terms of $t^{j}{ }_{i}$ and the spin form too. The exterior derivative of (2) above looks like as a conservation equation again, but Einstein's equations, in contrast to Theorem 3.2.1., can be recovered from this conservation equation only up to a non specified cosmological constant:

\section{Theorem 3.3.2.:}

Let $T_{i}=T^{j}{ }_{i} \Sigma_{j}$ be any horizontal $\mathbf{R}^{m *}$-valued $(m-1)$ form on $L(M)$, satisfying $d T_{i}-\omega^{e}{ }_{i} \wedge T_{e}=0$, $\kappa \in \mathbf{R}$ and $m \neq 2$. Then

(1') $\omega^{a}{ }_{b}$ is torsion free and $\exists \Lambda \in \mathbf{R}$ such that $G^{j}{ }_{i}+\Lambda \delta_{i}^{j}=\kappa T^{j}{ }_{i}$,

(3) $d\left(\kappa T^{j}{ }_{i} \Sigma+t^{j}{ }_{i}\right)=0$

are equivalent statements.

Proof:

To prove $\left(1^{\prime}\right) \rightarrow(3)$ it is enough to show that $d\left(\Lambda \delta_{i}^{j} \Sigma\right)=0$, but, because of the vanishing of the torsion, it follows from eq.(3.1.6.).

To prove the converse, first calculate the exterior derivative of $t^{j}{ }_{i}$. After a simple but rather long calculation we arrive at

$$
\begin{aligned}
-2 d t^{j}{ }_{i} & ={\Omega^{a}}^{a} \wedge\left(\delta_{i}^{j}\left(\omega^{e b}-\omega^{b e}\right) \wedge \Sigma_{a b}+2 g^{j e} \omega^{b}{ }_{i} \wedge \Sigma_{a b}+\right. \\
& \left.+\delta_{i}^{e}\left(\omega^{b j}-\omega^{j b}\right) \wedge \Sigma_{a b}+\left(\omega^{e j}+\omega^{j e}\right) \wedge \Sigma_{i a}\right)+ \\
& +\Xi^{e} \wedge\left(\delta_{i}^{j} \omega^{a}{ }_{c} \wedge \omega^{c b} \wedge \Sigma_{a b e}+\omega^{a}{ }_{i} \wedge\left(\omega^{b j}-\omega^{j b}\right) \wedge \Sigma_{a b e}+\right. \\
& \left.+\omega^{a}{ }_{b} \wedge\left(\omega^{b j}+\omega^{j b}\right) \wedge \Sigma_{i a e}\right) .
\end{aligned}
$$

Furthermore, because of the condition imposed on $T_{i}$ :

$$
\begin{aligned}
d\left(T^{j}{ }_{i} \Sigma\right) & =d\left(\theta^{j} \wedge T_{i}\right)=\Xi^{j} \wedge T_{i}-\omega^{j}{ }_{e} \wedge \theta^{e} \wedge T_{i}-\theta^{j} \wedge d T_{i}= \\
& =\Xi^{j} \wedge T_{i}-T^{e}{ }_{i} \omega^{j}{ }_{e} \wedge \Sigma+T^{j}{ }_{e} \omega^{e}{ }_{i} \wedge \Sigma,
\end{aligned}
$$

thus the condition (3) of Theorem 3.3.2. takes the form

$$
0=-2 d\left(\kappa T^{j}{ }_{i} \Sigma+t^{j}{ }_{i}\right)=-2 \kappa \Xi^{j} \wedge T_{i}+2 \kappa\left(T^{e}{ }_{i} \omega^{j}{ }_{e}-T^{j}{ }_{e} \omega^{e}{ }_{i}\right) \wedge \Sigma-2 d t^{j}{ }_{i} .
$$

Let $\left\{D_{m}{ }^{n}\right\}$ be the collection of fundamental vector fields associated to the Weyl basis of $g l(m, \mathbf{R})$. Recall [27] that $\left\{D_{m}{ }^{n}\right\}$ together with the standard horizontal vector fields $\left\{B\left(\delta_{a}\right)\right\}$ form a basis 
on $L(M)$, dual to $\left\{\omega^{a} b, \theta^{e}\right\}$; and hence $\omega^{a}{ }_{b}\left(D_{m}{ }^{n}\right)=\delta_{m}^{a} \delta_{b}^{n}$ and $\theta^{e}\left(D_{m}{ }^{n}\right)=0$. Taking the interior product of eq.(3.3.8.) first with $D_{m}{ }^{n}$ and with $D_{r}{ }^{s}$ and then contracting in $j$ and $r$ and in $m$ and $s$ we obtain:

$$
0=(2-m) \Xi^{e} \wedge \Sigma_{i b e} g^{b n} .
$$

For $m \neq 2$ this implies the vanishing of the torsion. Substituting $\Xi^{e}=0$ back to eq.(3.3.8.) and using (3.1.10) we have

$$
0=2\left(\left(R_{e}^{j}-\kappa T^{j}{ }_{e}\right) \omega^{e}{ }_{i}-\left(R^{e}{ }_{i}-\kappa T^{e}{ }_{i}\right) \omega^{j}{ }_{e}\right) \wedge \Sigma .
$$

Taking its interior product with $D_{m}{ }^{n}$ and contracting in $n$ and $i$ :

$$
R^{j}{ }_{i}-\frac{1}{m} R \delta_{i}^{j}=\kappa\left(T^{j}{ }_{i}-\frac{1}{m} T^{k}{ }_{k} \delta_{i}^{j}\right) .
$$

This equation can be rewritten in the following form:

$$
G^{j}{ }_{i}-\kappa T^{j}{ }_{i}=-\delta_{i}^{j}\left(\frac{1}{2 m}(m-2) R+\frac{\kappa}{m} T_{k}^{k}\right)=:-\delta_{i}^{j} \Lambda .
$$

But then, because of the contracted (second) Bianchi identity and the differential condition imposed on $T_{i}, \Lambda$ must be constant. $\varnothing$

In the rest of this paragraph we calculate the pull backs of condition (2) of Theorem 3.3.1. and its contravariant and dual forms.

If $\sigma^{e j}{ }_{i}$ is defined by $s^{*}\left(\sigma^{j}{ }_{i}\right)=: \sigma^{e j}{ }_{i} s^{*}\left(\Sigma_{e}\right)$ then

$$
\begin{aligned}
s^{*}\left(d \sigma^{j}{ }_{i}\right) & =d \sigma^{e j}{ }_{i} \wedge s^{*}\left(\Sigma_{e}\right)+\sigma^{e j}{ }_{i} s^{*}\left(d \Sigma_{e}\right)= \\
& =\left(E_{e}^{\mu} \partial_{\mu} \sigma^{e j}{ }_{i}+\sigma^{e j}{ }_{i} \omega_{k e}^{k}\right) s^{*}(\Sigma) .
\end{aligned}
$$

If $s$ is a coordinate section then

$$
s^{*}\left(d \sigma^{j}{ }_{i}\right)=\frac{1}{\sqrt{|g|}} \partial_{e}\left(\kappa \sqrt{|g|}{ }_{E} \sigma^{e j}{ }_{i} s^{*}(\Sigma),\right.
$$

while for a rigid section it is

$$
s^{*}\left(d \sigma^{j}{ }_{i}\right)=\kappa \nabla_{\mu}\left(A E \sigma^{\mu \alpha}{ }_{\beta} \vartheta_{\alpha}^{j} E_{i}^{\beta}\right) s^{*}(\Sigma) .
$$

The pull back of $t^{j}{ }_{i}$ is

$$
\begin{aligned}
s^{*}\left(t^{j}{ }_{i}\right) & =s^{*}\left(\theta^{j}\right) \wedge s^{*}\left(t_{i}\right)+\omega_{r s}^{j} s^{*}\left(\theta^{r}\right) \wedge s^{*}\left(\sigma^{s}{ }_{i}\right)= \\
& =\left(\tau^{j}{ }_{i}+\omega_{r s}^{j} \sigma^{r s}{ }_{i}\right) s^{*}(\Sigma) .
\end{aligned}
$$

For coordinate section this is just

$$
s^{*}\left(t^{j}{ }_{i}\right)=\kappa_{E} t^{j}{ }_{i} s^{*}(\Sigma),
$$

i.e. Einstein's pseudotensor again, while for a rigid section

$$
s^{*}\left(t^{j}{ }_{i}\right)=\left(\kappa_{A E} \theta^{\alpha}{ }_{\beta} \vartheta_{\alpha}^{j} E_{i}^{\beta}+\frac{1}{2}{ }_{G} \vee_{\beta}{ }^{\alpha \mu} \nabla_{\mu}\left(\vartheta_{\alpha}^{j} E_{i}^{\beta}\right)\right) s^{*}(\Sigma) .
$$


Thus, observing that $\partial_{\mu E} \sigma^{\mu \alpha}{ }_{\beta}=\partial_{\mu E} s^{\mu \alpha}{ }_{\beta}$ and ${ }_{a E} \sigma^{\mu \alpha}{ }_{\beta}={ }_{a E} s^{\mu \alpha}{ }_{\beta}$, the pull backs of condition (2) above along coordinate or rigid sections are the equations (2.3.8.) and (2.4.23.), respectively.

One can take the contravariant form of the spin form, $\sigma^{j i}:=\sigma^{j}{ }_{e} g^{e i}$, and its exterior derivative:

$$
-d \sigma^{j i}=\kappa T^{j i}+\Theta^{j i}
$$

where

$$
\Theta^{j i}:=t^{j i}+\left(\omega^{i e}+\omega^{e i}\right) \wedge \sigma^{j}{ }_{e} .
$$

The pull back of $\Theta^{j i}$ along a general section is

$$
s^{*}\left(\Theta^{j i}\right)=\left(\tau^{j i}+\omega_{r s}^{j} \sigma^{r s i}+\left(\omega_{r s}^{i} g^{s e}+\omega_{r s}^{e} g^{s i}\right) \sigma^{r j}{ }_{e}\right) s^{*}(\Sigma) .
$$

Thus for a coordinate section

$$
s^{*}\left(\Theta^{j i}\right)=\kappa_{E} \theta^{j i} s^{*}(\Sigma)
$$

while for a rigid section

$$
\begin{aligned}
s^{*}\left(\Theta^{j i}\right) & =s^{*}\left(t^{j}{ }_{e}\right) \eta^{e i}= \\
& =\left(\kappa_{A E} \theta^{\alpha \beta} \vartheta_{\alpha}^{j} \vartheta_{\beta}^{i}+\frac{1}{2} g^{\beta \rho}{ }_{G} \vee_{\rho}{ }^{\alpha \mu} \nabla_{\mu}\left(\vartheta_{\alpha}^{j} \vartheta_{\beta}^{i}\right)\right) s^{*}(\Sigma) .
\end{aligned}
$$

The calculations show that the pull backs of eq.(3.3.11.) along coordinate or rigid sections are eq.(2.3.15.) and (2.4.23.), respectively. Trivially, the pull backs of the antisymmetric part of eq.(3.3.11.) are the Belinfante-Rosenfeld equations.

For the sake of completeness, finally, let us consider the dual form of the spin form:

$$
\sigma^{j} e_{2} \ldots e_{m}:=\theta^{j} \wedge u_{e_{2} \ldots e_{m}} .
$$

Then

$$
-d \sigma^{j} e_{2} \ldots e_{m}=\kappa T^{j}{ }_{e_{2} \ldots e_{m}} \Sigma+\Theta^{j}{ }_{e_{2} \ldots e_{m}},
$$

where $T^{j} e_{2} \ldots e_{m}:=T^{j e} \varepsilon_{e e_{2} \ldots e_{m}}$ and

$$
\Theta^{j}{ }_{e_{2} \ldots e_{m}}:=\Theta^{j e} \varepsilon_{e e_{2} \ldots e_{m}}-\omega^{k}{ }_{k} \wedge \sigma^{j e} \varepsilon_{e e_{2} \ldots e_{m}} .
$$

The pull back of $\sigma^{j} e_{2} \ldots e_{m}$ along a coordinate section is

$$
s^{*}\left(\sigma^{j} e_{2} \ldots e_{m}\right)=-\frac{1}{2} \frac{1}{(m-1) !} \partial_{f}\left(|g| G^{j r e f}\right) \epsilon_{e e_{2} \ldots e_{m}} \epsilon_{r r_{2} \ldots r_{m}} d x^{r_{2}} \wedge \ldots \wedge d x^{r_{m}},
$$

and hence

$$
-s^{*}\left(d \sigma^{j} e_{2} \ldots e_{m}\right)=\frac{1}{2} \frac{1}{m !} \partial_{r} \partial_{s}\left(|g| G^{j r e s}\right) \epsilon_{e e_{2} \ldots e_{m}} \epsilon_{l_{1} \ldots l_{m}} d x^{l_{1}} \wedge \ldots \wedge d x^{l_{m}} .
$$

Therefore the pull back $s^{*}\left(\Theta^{j} e_{2} \ldots e_{m}\right)$ must yield the Landau-Lifshitz pseudotensor again. In fact, it is 


$$
\begin{aligned}
& s^{*}\left(\Theta^{j} e_{2} \ldots e_{m}\right)=s^{*}\left(\Theta^{j e}\right) \varepsilon_{e e_{2} \ldots e_{m}}-\kappa \Gamma_{k r E}^{k} \sigma^{r j e} \varepsilon_{e e_{2} \ldots e_{m}} s^{*}(\Sigma)= \\
& =\kappa_{L L} t^{j e} \varepsilon_{e e_{2} \ldots e_{m}} s^{*}(\Sigma),
\end{aligned}
$$

and therefore the pull back of eq.(3.3.15.) along a coordinate section is equivalent to

$$
-\kappa \partial_{\mu}\left(|g|_{E} \sigma^{\mu \alpha \beta}\right)=|g|\left(G^{\alpha \beta}+\kappa_{L L} t^{\alpha \beta}\right) .
$$

Thus ${ }_{L L} \sigma^{\mu \alpha \beta}:={ }_{E} \sigma^{\mu \alpha \beta}$ plays the role of the spin pseudotensor in the Landau-Lifshitz case also. Because of the symmetry of $G^{\alpha \beta}$ and ${ }_{L L} t^{\alpha \beta}$, the algebraic Belinfante-Rosenfeld equation is simply $\partial_{\mu}\left(|g|{ }_{L L} \sigma^{\mu[\alpha \beta]}\right)=0$. The Belinfante-Rosenfeld combination of these pseudotensors is essentially tensorial again:

$$
\begin{aligned}
|g|{ }_{L L} t^{\alpha \beta} & +\partial_{\mu}\left(|g|\left({ }_{L L} \sigma^{\mu[\alpha \beta]}+{ }_{L L} \sigma^{\alpha[\beta \mu]}+{ }_{L L} \sigma^{\beta[\alpha \mu]}\right)\right)= \\
& =|g|{ }_{L L} t^{\alpha \beta}+\partial_{\mu}\left(|g|{ }_{L L} \sigma^{\mu(\alpha \beta)}\right)= \\
& =-\frac{1}{\kappa}|g| G^{\alpha \beta} .
\end{aligned}
$$

The Noether pseudocurrent, associated to a vector field $K^{\mu}$, is defined by

$$
\begin{aligned}
{ }_{L L} C^{\mu}[\mathbf{K}]: & ={ }_{L L} t^{\mu \nu} K_{\nu}+\left({ }_{L L} \sigma^{\mu[\alpha \beta]}+{ }_{L L} \sigma^{\alpha[\beta \mu]}+{ }_{L L} \sigma^{\beta[\alpha \mu]}\right) \partial_{\alpha} K_{\beta}= \\
& ={ }_{L L} t^{\mu \nu} K_{\nu}+{ }_{L L} \sigma^{\mu \alpha \beta} \partial_{\alpha} K_{\beta}= \\
& =-\frac{1}{\kappa} G^{\mu \nu} K_{\nu}+\frac{1}{|g|} \partial_{\alpha}\left(|g|{ }_{L L} \sigma^{\mu \alpha \beta} K_{\beta}\right) .
\end{aligned}
$$

Then $|g|\left(\kappa_{L L} C^{\mu}[\mathbf{K}]+G^{\mu \nu} K_{\nu}\right)$ is always pseudoconserved. However, if $\mathbf{K}$ is a Killing vector of the geometry then, in general, it is not the sum of separately (pseudo)conserved matter and gravitational pseudocurrents: We have only

$$
\begin{aligned}
0 & =\partial_{\mu}\left(|g|\left(\kappa_{L L} C^{\mu}[\mathbf{K}]+G^{\mu \nu} K_{\nu}\right)\right)= \\
& =|g| \frac{1}{2} G^{\mu \nu} \mathrm{E}_{\mathbf{K}} g_{\mu \nu}+ \\
& +\kappa \partial_{\mu}\left(|g|{ }_{L L} C^{\mu}[\mathbf{K}]\right)+|g| G^{\mu \nu} K_{\nu} \Gamma_{\mu \rho}^{\rho},
\end{aligned}
$$

and hence the gravitational Noether pseudocurrent is not pseudoconserved even for a Killing vector $\mathbf{K}$. For vector fields satisfying $\partial_{(\alpha} K_{\beta)}=0$ the pseudocurrent $|g|\left(\kappa_{L L} C^{\mu}[\mathbf{K}]+G^{\mu \nu} K_{\nu}\right)$ is the sum of two separately pseudoconserved parts: the first, as we saw at the end of the previous paragraph, is $|g|\left(\kappa_{L L} t^{\mu \nu}+G^{\mu \nu}\right) K_{\nu}$; and the second is $|g|{ }_{L L} \sigma^{\mu \alpha \beta} \partial_{\alpha} K_{\beta}$. However, if $K_{\nu}$ generates coordinate rotation then the second part is not zero, and, if we accepted the interpretation of ${ }_{L L} \sigma^{\mu \alpha \beta}$ as the spin pseudotensor of gravity in the Landau-Lifshitz formulation - which view would be supported by the interpretation of $\sigma^{j}{ }_{i}$ as the spin form and the fact that the relation between ${ }_{L L} \sigma^{\mu \alpha \beta}$ and ${ }_{L L} t^{\alpha \beta}$ is the same that between e.g. ${ }_{E} \sigma^{\mu \alpha \beta}$ and ${ }_{E} \theta^{\alpha \beta}-$, the result would be rather surprising: the orbital and spin angular momenta of gravity would be separately conserved.

This strange behaviour, together with others mentioned above and at the end of the previous paragraph, supports our view that the proper energy-momentum and spin pseudotensors of gravity are the canonical ones.

\subsection{The Noether form on $L(M)$}


Let $\mathbf{K}$ be any vector field on $M$ and $\left\{K^{a}\right\}$ be the collection of functions on $L(M)$ defined by $\mathbf{K}$ : if $w=\left(p,\left\{\mathbf{E}_{a}\right\}\right) \in L(M)$ then let $K^{a}(w)$ be the ath component of $\mathbf{K}$ in the basis $\left\{\mathbf{E}_{a}\right\}$ at $T_{p} M$. (In the language of Kobayashi and Nomizu [27] $\left\{K^{a}\right\}$ is a zero form on $L(M)$ of type $\left(\mathbf{R}^{m}, G L(m, \mathbf{R})\right)$.) Thus $\mathrm{L}_{D_{m}{ }^{n}} K_{a}=\delta_{a}^{n} K_{m}$, where $D_{m}{ }^{n}$ is the fundamental vector field on $L(M)$ associated to the element $e_{m}{ }^{n}$ of the Weyl basis of $g l(m, \mathbf{R}) . \mathbf{K}$ is a conformal Killing vector on $M$ iff $\mathrm{E}_{B\left(\delta_{a}\right)} K_{b}+\mathrm{E}_{B\left(\delta_{b}\right)} K_{a}=\Omega g_{a b}$ for some $G L(m, \mathbf{R})$-invariant function $\Omega$ on $L(M)$; and $\mathbf{K}$ is a Killing vector iff $\Omega=0$. Here $\left\{B\left(\delta_{a}\right)\right\}$ are the standard horizontal vector fields on $L(M)$.

The gravitational Noether form, associated to $\mathbf{K}$, is defined by

$$
\begin{aligned}
C[\mathbf{K}]: & =\Theta^{a} K_{a}+d K_{a} \wedge u^{a}= \\
& =\Theta^{a} K_{a}+K_{e} \omega^{e}{ }_{a} \wedge u^{a}+\mathrm{E}_{B\left(\delta_{a}\right)} K_{b} \sigma^{a b} .
\end{aligned}
$$

Then trivially

$$
d\left(K_{a} u^{a}\right)=C[\mathbf{K}]+\kappa T^{a} K_{a},
$$

and the pull back of $C[\mathbf{K}]$ along a general local section of $L(M)$ is

$$
\begin{aligned}
s^{*}(C[\mathbf{K}]) & =s^{*}\left(\Theta^{a}\right) K_{a}+s^{*}\left(d K_{a}\right) \wedge s^{*}\left(u^{a}\right)= \\
& =s^{*}\left(\Theta^{a}\right) K_{a}+E_{a}^{\mu} \partial_{\mu} K_{b} s^{*}\left(\sigma^{a b}\right) .
\end{aligned}
$$

If $s$ is a coordinate section then

$$
s^{*}(C[\mathbf{K}])=\kappa\left({ }_{E} \theta^{\mu \nu} K_{\nu}+{ }_{E} \sigma^{\mu \alpha \beta} \partial_{\alpha} K_{\beta}\right) s^{*}\left(\Sigma_{\mu}\right),
$$

which is just the Noether pseudocurrent (2.3.17.) built up from the Einstein pseudotensors in holonomic description. For a rigid section the pull back is

$$
\begin{aligned}
s^{*}(C[\mathbf{K}]) & =\kappa\left({ }_{a E} \theta^{\mu \nu} K_{\nu}+{ }_{a E} \sigma^{\mu \alpha \beta} \partial_{\alpha} K_{\beta}\right) s^{*}\left(\Sigma_{\mu}\right)= \\
& =\kappa\left({ }_{A E} \theta^{\mu \nu} K_{\nu}+{ }_{A E} \sigma^{\mu \alpha \beta} \nabla_{\alpha} K_{\beta}\right) s^{*}\left(\Sigma_{\mu}\right),
\end{aligned}
$$

the Noether current (2.4.17.), (2.4.26.). Thus although the pull backs of the Sparling type forms $t_{i}, t^{j}{ }_{i}$ and $\Theta^{i}, \Theta^{j i}$ along rigid sections are not exactly the tensors ${ }_{A E} \theta^{j}{ }_{i}$ and ${ }_{A E} \theta^{j i}$, respectively, the pull backs of the Noether form are the Noether (pseudo)currents. The Noether form is therefore seems to be the geometric object on $L(M)$ which, with appropriately chosen vector field $\mathbf{K}$, describes the momentum-angular momentum content of gravity.

A simple consequence of eq. (3.4.2.) is $d\left(C[\mathbf{K}]+\kappa T^{a} K_{a}\right)=0$; and since

$$
\begin{aligned}
d C[\mathbf{K}] & =-\kappa\left(d K_{a} \wedge T^{a}+K_{a} d T^{a}\right)= \\
& =-\kappa \mathrm{E}_{B\left(\delta_{a}\right)} K_{b} T^{a b} \Sigma,
\end{aligned}
$$

and the symmetry $T^{a b}=T^{(a b)}$, implied by the symmetry of Einstein's tensor in absence of torsion, $C[\mathbf{K}]$ is closed for Killing vectors.

\section{Summary and discussion}

In the present paper the "orthodox" pseudotensorial description of the gravitational linear and angular momenta was reexamined. In the usual approach three difficulties are involved: (1) There 
is an ambiguity in the definition of the conserved quantities: one can add to them arbitrary curls, and it does not seem to have a selection rule to rule out this ambiguity. With an appropriate curl one can obtain the completely different energy-momentum expressions of Einstein, Bergmann, Møller, Komar etc. (2) In contrast to the requirement of the principle of general covariance the conserved quantities are in general not geometric objects. Although Komar's expression is tensorial, but at infinity for the Kerr spacetime it yields $2 \frac{a}{m}$ as the angular momentum/mass ratio instead of the expected $\frac{a}{m}$ [8]. In the " $m$-legs" formalism of gravity the conserved quantities are all $S O(p, q)$-gauge dependent. (3) The conserved quantities are associated to vector fields on the spacetime manifold. Thus they are built up not only from the gravitational field variables, but the actual vector field too. Therefore there are infinitely many conserved quantities even if the superpotential is fixed. One wants to identify $m$ of them as the linear, and $\frac{1}{2} m(m-1)$ as the angular momentum. However it is not a priori clear how to choose the corresponding vector fields and what is the interpretation of the remaining infinite conserved quantities.

In the first part of this paper the first difficulty was tried to resolve. To choose from the mathematically possible infinitely many conserved currents and pseudotensors we followed the scenario of the Lagrangian theory of matter fields. However, at least in well known textbooks on field theory, this formalism is available only for first order Lagrangians. Since we wanted to consider not only first order, but second order Lagrangian too, first we had to generalize the formalism. This has been done in paragraph 2.1 .

In contrast to the canonical energy-momentum and spin tensors the symmetric energy-momentum tensor has several attractive properties, e.g. gauge invariance, independence of total divergences added to the Lagrangian, and its contraction with any Killing vector is conserved if the field equations are satisfied. Thus the symmetric energy-momentum tensor seems to be better to describe the energy-momentum and angular momentum of fields. However, conceptually the notion of energy, momentum and angular momentum is connected to the first Noether theorem. In fact, this theorem, applied for the material action, gives us just the identity (2.1.10.) and predicts the conservation of the Noether current (2.1.14.), provided $\mathbf{K}$ is a Killing vector and the field equations are satisfied. On the other hand, the interpretation of the symmetric energy-momentum tensor as the relativistic energy-momentum density of fields is possible only since it is the BelinfanteRosenfeld combination of the canonical tensors. One may say that this interpretation is a matter of taste. However, this can be so until gravitation is taken into account. In fact, there is no gravitational counterpart of the symmetric energy-momentum tensor of the matter fields, even in principle, while one can apply the first Noether theorem for the total action and any vector field and obtain conserved Noether currents. The symmetric energy-momentum tensor of the matter fields is simply the "source density of gravitation".

Unfortunately, as we saw at the beginning of paragraph 2.2., we cannot introduce acceptable tensorial energy momentum and angular momentum expressions for gravity, and hence, for lack of better, we should use the so-called pseudotensorial quantities. Since we would like to have as complete characterization of the energy, momentum and angular momentum properties of gravity as it is possible, we had to consider the gravitational counterpart not only of the canonical energy-momentum, but the spin tensor as well. These pseudotensors were defined for second order Lagrangians in the rest of paragraph 2.2 .

Paragraphs 2.3., 2.4. and 2.5. are systematic applications of the general Lagrangian scenario. First the metric was chosen as the gravitational field variable and Einstein's first order Lagrangian 
was considered. It was shown that the spin pseudotensor plays the role of a superpotential for the energy-momentum pseudotensor and von Freud's superpotential is distinguished among the superpotentials by the Lagrangian formalism. The contravariant form of these pseudotensors were defined and it was shown that they satisfy the Belinfante-Rosenfeld equations. Furthermore, their Belinfante-Rosenfeld combination is just Einstein's tensor. The Noether pseudocurrent, defined in the standard way, was shown to give the angular momentum expression of Bergmann and Thomson as a special case.

Then, instead of the metric, a rigid basis of 1-forms was chosen as the field variable. The spin pseudotensor, apart from the factor $\sqrt{|g|}$, was shown to be tensorial, just minus the superpotential of Goldberg. A tensorial canonical energy-momentum expression was found, such that these tensors satisfy the tensorial Belinfante-Rosenfeld relations. The Noether current constructed from them is also tensorial. However, they are not invariant with respect to local rigid rotations of the basis 1 -forms.

Finally, Hilbert's second order Lagrangian was considered. It was found that the canonical energy-momentum pseudotensor is nothing but Møller's energy-momentum pseudotensor, introduced originally in a completely different way. The spin pseudotensor plays also the role of a superpotential and distinguishes Møller's superpotential. The results do not depend on whether the field variable is the metric or a rigid basis of 1-forms. (In the previous two cases not only the description, but the Lagrangian was different too.)

Now turn to the problem of the appearing contradiction to the principle of general covariance. As is usually stated the principle of general covariance requires that the laws of Nature must have a form containing only geometric objects. In the holonomic description the Noether pseudocurrents are not tensorial objects, in contrast to the requirement of the principle of general covariance. Although for first order Lagrangian and anholonomic description the Noether current is a true vector field, but it depends on the basis 1-forms; i.e. it is $O(p, q)$-gauge dependent. The stronger form of the principle of general covariance formulated e.g. in [1] does not allow this gauge dependence either.

However, if the geometric objects in the principle of general covariance were not required to be geometric objects on the spacetime manifold, but they were allowed to be geometric objects on the manifold of frames of the spacetime; i.e. on the bundle of linear frames $L(M)$ over $M$, and if the previous coordinate and/or gauge dependent quantities and formulae could be reformulated in terms e.g. of differential forms on $L(M)$, then the contradiction with the principle of general covariance would be resolved. This reformulation of the canonical pseudotensors for the first order gravitational Lagrangian was done in the second part of this paper.

First we defined the Nester-Witten and Sparling forms on the bundle of linear frames (as Frauendiener did in four dimensions [32]) in $m$ dimensions (as Dubois-Violette and Madore did on the bundle of orthonormal frames [31]), and then their contravariant and dual forms were introduced. Frauendiener has shown that the pull back of Sparling's form along a coordinate section of $L(M)$ is Einstein's canonical energy-momentum pseudotensor, and the pull back of the contravariant form of Sparling's form was tried to identify as the Landau-Lifshitz pseudotensor $[32,33]$. Here we showed that the pull back (along a coordinate section) of the contravariant form of Sparling's form is Bergmann's pseudotensor, while the Landau-Lifshitz pseudotensor can be recovered as the pull back of the dual form of Sparling's form. The pull backs of Sparling's form and its contravariant form were calculated along rigid sections of $L(M)$ too. It turned out 
that although the results are tensorial but neither the canonical energy-momentum tensor nor its contravariant form is a simple pull back of some form of Sparling's form. However, the pull backs of the full Sparling equation (or its contravariant form) always yield the equations giving the canonical energy-momentum tensors in terms of the corresponding superpotentials.

Then a $g l(m, \mathbf{R})$-valued $(m-1)$ form, the so-called spin form and its contravariant form were defined. It is known that by means of Sparling's form one can give necessary and sufficient conditions for a metric connection being torsion free and solution of Einstein's equations. It was proved that similar equivalent characterization of Einstein's theory is possible by means of the spin form too. The pull back of the spin form and its covariant form along any section are shown to be the corresponding canonical spin pseudotensor. Exterior differential equations for the various forms of the spin form could be derived, the pull backs of whose antisymmetric part are always the Belinfante-Rosenfeld equations for the (pseudo) tensors. Finally, for any vector field of the spacetime an $(m-1)$ form, called the Noether form, was defined on $L(M)$, whose pull backs are always the corresponding Noether pseudocurrents.

The comparison of the apparently different and complicated pseudotensorial calculations with the simple differential form approach suggests to consider the bundle of linear frames as more natural arena to describe the gravitational (and, to retain the unity of physics, the physical) phenomena than the spacetime itself. Although it has been shown only in the holonomic and anholonomic descriptions that the pseudotensorial formulae and quantities can be recovered from Sparling's equation and differential forms on $L(M)$, respectively, we interpret the spin form (it is only a name here), the Sparling form and the Nester-Witten form as the spin and energymomentum form of gravity, and the superpotential for the energy-momentum form, respectively.

Accepting this interpretation and recalling that the Landau-Lifshitz pseudotensor is a pull back of the dual form of Sparling's form along a coordinate section, the pull back of the dual form of the spin form would have to be interpreted as the spin pseudotensor in the Landau-Lifshitz approach. (Since the Landau-Lifshitz energy-momentum pseudotensor is not a canonical pseudotensor, one cannot use the Lagrangian scenario to construct the corresponding spin pseudotensor.) However, it turned out that the orbital and spin parts of the total Landau-Lifshitz angular momentum are separately conserved. This might suggest not to consider the Landau-Lifshitz pseudotensors as the proper quantities describing the energy-momentum and angular momentum properties of gravity.

Unfortunately, we could not recover Møller's superpotential, pseudotensors and Noether pseudocurrent as pull backs of certain differential forms on $L(M)$ such that, at the same time, the pull backs of the exterior differential equations for them would be the familiar pseudotensorial equations.

The reformulation of the pseudotensorial formalism in the coordinate free differential geometric language does not mean, of course, that the notion of gravitational energy-momentum and angular momentum became coordinate (gauge) independent. Mathematically this gauge dependence is coded in the non horizontal character of the Nester-Witten, Sparling, spin and the Noether forms, and hence their pull backs along local sections of $L(M)$ will depend on the actual sections. (In the language of [27] these forms are only pseudotensorial, but not tensorial.)

The gauge dependence of the Noether current is not a specific property of gravity, it is common in non abelian gauge theories: In the gauge theory specified at the end of paragraph 2.1. the field equation for the connection fields is 


$$
-J_{\Gamma}^{\mu}=2 \nabla_{\nu}\left(\frac{\partial L_{c}}{\partial F_{\mu \nu}^{\Gamma}}\right)+2 \frac{\partial L_{c}}{\partial F_{\mu \nu}^{\Gamma}} c_{\Gamma \Pi}^{\Delta} A_{\nu}^{\Pi},
$$

where $J_{\Gamma}^{\mu}$, the "source density of the connection fields", is defined as the functional derivative of the particle action with respect to $A_{\mu}^{\Gamma}$. Denoting the second term on the right by $j_{\Gamma}^{\mu}$, the connection field equation is equivalent to

$$
J_{\Gamma}^{\mu}+j_{\Gamma}^{\mu}=-2 \nabla_{\nu}\left(\frac{\partial L_{c}}{\partial F_{\mu \nu}^{\Gamma}}\right) .
$$

While $J_{\Gamma}^{\mu}$ is gauge covariant, neither $j_{\Gamma}^{\mu}$ nor the right hand side, the superpotential for the conserved currents, is gauge covariant. This is the reason why $j_{\Gamma}^{\mu}$ is sometimes called pseudocurrent. Since it is proportional to the structure constant of $G$, it vanishes for abelian $G$.

If, however, the notion of gravitational energy-momentum and angular momentum is necessarily gauge dependent, one may ask whether it is good for anything at all, and the problem considered here is not only a rule how to split the gauge independent Einstein tensor into energymomentum and spin parts in a gauge dependent way. Isn't it a pure gauge? It would be difficult to avoid this question if we wanted to have only local, energy density-like quantities. However, if we have a closed $(m-2)$ dimensional closed submanifold $\mathcal{S}$ in $M$ then, in contrast to internal gauge theories, $\mathcal{S}$ may be used to reduce the gauge freedom at the points of $\mathcal{S}$. Thus although we use the non-horizontal Nester-Witten form, the integral of its pull backs to $\mathcal{S}$ along the preferred local sections may be well defined quasi local integrals. These quasi local integrals will be considered elsewhere.

Finally, in this paper we did not deal with difficulty (3). The vector field $\mathbf{K}$, if it was specified at all, was defined by certain properties of the coordinate transformation it generated. It does not seem to be possible to specify $\mathbf{K}$ geometrically within the framework considered here. Quasi locally, however, there is a chance to define $\mathbf{K}$ to generate acceptable linear and angular momenta of the matter + gravity system.

\section{Acknowledgments}

The author is grateful to Professors Peter Bergmann and Venzo De Sabbata for the useful discussions on the spin of gravity, and to the Ettore Majorana Centre for the financial support during the 12th Erice Summer School of Cosmology and Gravitation, where a part of this paper could be presented.

\section{References}

[1] R. M. Wald, General relativity, The University of Chicago Press, 1984

[2] S. W. Hawking, G. F. R. Ellis, The large scale structure of spacetime, Cambridge Univ. Press, Cambridge 1973

[3] S. Weinberg, Gravitation and Cosmology, John Wiley and Sons Inc. New York, 1972

[4] A. Trautman, Conservation Laws in Gen. Rel., in Gravitation: An Introduction to Current Research, Ed.: L. Witten, Wiley, New York 1962 
[5] A. Trautman, F. A. E. Pirani and H. Bondi, Lectures on General Relativity, Brandeis Summer Institute in Theor. Phys., New Jersey 1964

[6] P. G. Bergmann, The general theory of relativity, in Handbuch der Physik IV., Ed.: S. Flugge, Springer - Verlag, Berlin 1962

[7] J. Horsky and J. Novotny, Czech. J. Phys.B 19419 (1969)

[8] J. N. Goldberg, Invariant transformations, conservation laws, and energy-momentum, in: General relativity and gravitation, Vol.1, Ed.: A. Held, Plenum, New York, 1980

[9] P. G. Bergmann, Phys. Rev. 112287 (1958)

[10] A. J. Anderson: Principles of Relativity Physics, Acad. Press, New York and London, 1967, Ch. 13

[11] F. J. Belinfante, Physica, VI. 887 (1939)

[12] F. J. Belinfante, Phisica, VII. 305 (1940)

[13] L. Rosenfeld, Mem. Roy. Acad. Belg. Cl. Sci. 18 No6 (1940)

[14] F. W. Hehl, Rep. Math. Phys. 955 (1976)

[15] F. W. Hehl, P. von der Heyde, G. D. Kerlick, J. M. Nester, Rev. Mod. Phys. 48393 (1976)

[16] R. Penrose, W. Rindler, Spinors and spacetime Vol 1, Spinor calculus and relativistic fields, Cambridge Univ. Press, Cambridge, 1984

[17] R. Utiyama, Phys. Rev. 1011597 (1956)

[18] F. H. J. Cornish, Proc. Roy. Soc. Lond. A 282358 (1964)

[19] M. Ferraris, M. Francaviglia, Gen. Rel. Grav. 22965 (1990)

[20] C. Møller, Ann. Phys. 4347 (1958)

[21] A. Komar, Phys. Rev. 113934 (1959)

[22] F. de Felice, C. J. S. Clarke, Physics in curved manifolds, Cambridge Univ. Press, Cambridge, 1990

[23] P. G. Bergmann, R. Thomson, Phys. Rev. 89400 (1953)

[24] J. N. Nester, Phys. Lett. 139 A 112 (1989)

[25] J. N. Goldberg, Phys. Rev. 111315 (1958)

[26] L. Landau, E. M. Lifshitz, Classical theory of fields, (in Hungarian), Tankönyvkiadó, Budapest 1975

[27] S. Kobayashi, K. Nomizu, Foundations of Differential Geometry, Vol 1, Interscience 1964

[28] G. A. J. Sparling, Twistors, spinors and the Einstein vacuum equations, Preprint, 1982

[29] M. Dubois-Violette, J. Madore, Commun. Math. Phys. 108213 (1987)

[30] J. N. Nester, Phys. Lett. 83 A 241 (1981)

[31] E. Witten, Commun. Math. Phys. 80381 (1981)

[32] J. Frauendiener, Class. Quantum Grav. 6 L237 (1989)

[33] L. J. Mason, J. Frauendiener, in Twistors in Mathematics and Physics, London Math. Soc. Lecture Notes No. 156, Ed.: T. N. Bailey and R. J. Baston, Cambridge Univ. Press, Cambridge 1990 